\title{
10 DER KRIEGER UND DIE KAISERIN
}

Im vorangegangenen zweiten Teil der Arbeit wurde die Idee des Metaphorisierens in Auseinandersetzung mit einem klassischen Hollywoodfilm entwickelt. Mit Tom Tykwers DER KRIEgER UND DIE KAISERIN (D 2000) wende ich mich nun mit jener Perspektive auf die Konstitution von Bedeutung einem zeitgenössischen deutschen Spielfilm zu. Dabei handelt es sich um einen sehr komplexen Film, in dem sprachliche Einfachheit und inszenatorische Virtuosität, Direktheit und Mehrdeutigkeit unauflösbar miteinander verschmelzen.

Auf narrativer Ebene betrachtet, erzählt DER KRIEGER UND DIE KAISERIN die Geschichte der in der geschlossenen psychiatrischen Anstalt geborenen, wohnenden und arbeitenden Krankenschwester Simone „Sissi“ Schmidt (Franka Potente), deren Leben bei einem schweren Autounfall vom Ex-Soldaten Bodo Riemer (Benno Führmann) gerettet wird. Sissi verliebt sich dabei in Bodo und sieht im Zusammensein mit ihm die Möglichkeit, ihre hermetische Psychiatriewelt und die an ihr emotional hängenden und oft dominierenden Patienten verlassen und „draußen glücklich“ werden zu können. Doch Bodo - nach dem gewaltsamen Verbrennungstod seiner Frau aufgrund einer Tankstellenexplosion traumatisiert und beziehungsunfähig - blockt zunächst ihre Versuche, Kontakt mit ihm aufzunehmen, brutal ab. Sissis und Bodos Wege kreuzen sich in der Folge erneut bei einem Bankraub, den Bodo mit seinem Bruder Walter (Joachim Król) begeht, um mit ihm nach Australien auswandern zu können, und bei dem Walter tödlich verletzt wird. Sissi, die ebenfalls an diesem Tag in der Bank ist, kommt hier nun Bodo zu Hilfe. Schließlich - zum Ende des Films - gelingt es beiden dann gemeinsam, ihr jeweiliges altes Leben hinter sich zu lassen und zusammen zu sein.

Nicht nur der Titel des Films ist dabei märchenhaft. Auch die Liebessehnsucht Sissis bedient sich auf ganz eigene Weise der Romantik klassischer und moderner Märchenerzählungen. So vermischen sich Dornröschen und Schneewittchen in dem langen, schier ,ewig' dauernden Moment, in dem Sissi (auf die frontal ein Lastwagen in voller Fahrt aufgeprallt ist) fast wie in einem Sarg unter dem Lastwagen liegt, ohne atmen zu können, und Bodo ihr schließlich mit einem Luftröhrenschnitt das Leben rettet. Und wenn daraufhin Sissi nur noch den Knopf von Bodos Jackenärmel in der Hand behält, bevor sie ihren Lebensretter aus den Augen verliert, und sie sich im weiteren Verlauf des Films dann mit diesem Knopf auf die Suche nach Bodo macht und ihn damit identifiziert, ist das rollenvertauschte Aschenputtel-Motiv offensichtlich.

Später sieht man, wie die unglückliche, zurückgewiesene Sissi Vittorio de Sicas neorealistisches Märchen das WUNDER von mailand (Miracolo a milano, I 1951) im Fernsehen schaut: eine schließlich vor Glück berstende Szene zwischen

Ә Open Access. ( 2020 Christina Schmitt, publiziert von De Gruyter. (C) BY-NC-ND Dieses Werk ist lizenziert unter der Creative Commons Attribution-NonCommercial-NoDerivatives 4.0 Lizenz. https://doi.org/10.1515/9783110614619-010 
einem jungen Mann und einem jungen Mädchen - strahlende Gesichter, Küsse und eine aufgehende Sonne, die der Mann dem Mädchen schenkt.

Das Märchenhaft-Schwebende dieses Films, der in seinen Inszenierungsweisen ebenso mit dem Melodramatischen arbeitet wie mit Action, Suspense und Horror (und auf ganz eigene Weise vielfach Bezug nimmt auf Miloš Formans Psychiatrie-Drama ONE FLEW OVER THE CUCKOo's NEST [USA 1975]) ${ }^{1}$ geht nun jedoch längst nicht allein aus solchen assoziativen Motiven und dem Happy End hervor. Vielmehr liegt es an seiner gesamten, all solche Aspekte sich spezifisch aneignenden Machart, die im Sehen und Hören eine phantastische Welt entstehen lässt, die voller elementarer, hyperintensiv wirkender Kräfte ist, und in der zwei auf je eigene Weise verletzte, Abstand suchende, isolierte Menschen miteinander verbunden sind, schon lange bevor sie sich zum Schluss an die Hand nehmen, ein Paar werden und zusammen weggehen. Aufs Ganze betrachtet, emergieren im Verlauf der zwei Stunden von DER KRIEGER UND DIE KAISERIN durch ein vielfach sich verzweigendes, durch Wiederholungen und Variationen sich bewegendes Metaphorisieren zwei große systematische Metaphern. Man erfährt Liebe als die Schwierigkeit, sich zu berühren, ohne sich zu verletzen. ${ }^{2}$ Und die zweite große Metapher, die entsteht, kann tentativ mit der Formulierung Seiner Bestimmung folgen ist Veränderung gefasst werden. Dies sei im nächsten Abschnitt zunächst makroanalytisch skizziert, bevor einige Aspekte hiervon mikroanalytisch vertieft werden.

\section{Liebe ist die Schwierigkeit, sich zu berühren, ohne sich zu verletzen - und: Seiner Bestimmung folgen ist Veränderung. Skizze zweier systematischer Metaphern}

Der Erfahrungsbereich der Liebe wird mit verschiedenen Beziehungsformen ausgearbeitet: Im Zentrum steht die Liebe zwischen Mann und Frau (zwischen Sissi und ihrem Patienten Steini [Lars Rudolph], zwischen Bodo und seiner gewaltsam getöteten Frau sowie zwischen Sissi und Bodo), aber auch geschwisterlichfreundschaftliche Liebe (zwischen Sissi und ihrem Patienten Otto [Melchior Beslon], zwischen Bodo und seinem Bruder) oder die weitere familiäre Liebe (zwi-

\footnotetext{
1 Sowohl im Anschluss an Lynne Camerons Dynamic-Discourse-Ansatz als auch im Anschluss an die Genretheorie Hermann Kappelhoffs (vgl. Kappelhoff: Genre und Gemeinsinn) impliziert die Auffassung eines Films als einem Diskursereignis, das für sich betrachtet werden kann, immer auch schon dessen Geschichtlichkeit: im Sinne einer Geschichtlichkeit von Wahrnehmungsformen.

2 Ich danke Hermann Kappelhoff für den Austausch hierzu.
} 
schen Sissi und den anderen Patienten) wird verhandelt. In Korrelation wird dieser Erfahrungsbereich der Liebe wiederum mit Berührungsformen gebracht, die in ihren Qualitäten ganz unterschiedlich und zumeist extrem sind. Die Vielzahl an Berührungsformen sei im Folgenden skizziert - und dabei ist immer im Blick zu behalten, dass mit diesen hier sehr kondensierten Skizzen immer schon von audiovisuellen Wahrnehmungsakten die Rede ist, die man als Zuschauer durchläuft, und dass erst diese Wahrnehmungsverschränkung so etwas wie das Figurenerleben evoziert.

So gibt es sanfte Berührungen (das Ertasten von Sissis Gänsehaut durch den Patienten Otto, das Streichen über die Wange einer im offenen Sarg liegenden toten alten Frau durch Bodo, das Berühren von Bodos Schulter durch Sissi), doch genauso gibt es brutale Berührungen (der Schwerlaster, Bodo und der Patienten Werner [Ludger Pistor], die Sissi auf je unterschiedliche Weisen zu Boden stoßen und schlagen) sowie erzwungene Berührungen (das Tanzen mit und die manuelle Befriedigung von Steini durch Sissi, wie unten analysiert). Immer wieder überschreiten diese Berührungen Grenzen: Sie sind $\mathrm{zu}$ hart und bringen einen mit ihrer tödlichen Gewalt fast um (der auf Sissi aufprallende Schwerlaster), stoßen einen zurück, obwohl man versucht, in Kontakt zu kommen (Bodos Niederboxen Sissis). Sie sind $\mathrm{zu}$ heiß, man kann sich daran verbrennen (Bodos zwanghaftes Umarmen eines brennenden Ofens) - und mehr noch: das Verbrennen (die traumatische Tankstellen-Explosion, ein Krematorium) kann letztlich alles vernichten (hierauf wird mikroanalytisch zurückgekommen). Kühlung kann Linderung verschaffen (mit Eis und Wasser), oder das Gewinnen von Abstand (Walters Plan, mit Bodo nach Australien, auf die andere Seite der Erde, auszuwandern, Walters Aufforderung an Bodo, „vom Klo runter“ zu kommen ${ }^{3}$ ). Eine andere Grenzüberschreitung ist, dass Körper einem zu nah kommen, dominierend an einem hängen - und es schwer ist, hier Distanz und damit Raum für sich selbst, auch für das eigene Begehren finden zu können (die Arbeit von Sissi als Krankenschwester in der Psychiatrie). Erst am Ende findet sich im An-die-HandNehmen von Sissi und Bodo ein angemessener Berührungsmodus: eine Berührung, die Kontakt und Sicherheit in der Welt jenseits der Psychiatrie ermöglicht und zugleich auch genug Abstand zulässt.

Ein in Variationen wiederholtes expressives Muster, mit dem Verbrennen, Hitze bzw. große Wärme thematisiert werden, ist der die Zuschauerwahrnehmung modellierende filmische Ausdruck eines langsamen Sogs, dessen Bindungskraft

3 Das Immer-noch-auf-dem-Klo-Sein Bodos ist die sprachliche Metapher, mit der Walter Bodos Gefangensein in der traumatischen Erfahrung beschreibt: in dem Verbrennungstod seiner Frau, während er auf dem Klo der dann explodierenden Tankstelle war. 
zuletzt konterkariert wird (hierauf wird zurückzukommen sein). Und die Sensitivität für das Spüren aller oben skizzierten Berührungen im Sehen und Hören des Films wiederum liegt in dessen Kinematografie selbst, die das Spüren, das sinnliche Wahrnehmungsvermögen als Erfahrung immer wieder ausstellt, sinnlichsynästhetisch anschaulich macht: so beispielsweise das Spüren von Gänsehaut (s. u.) oder das Hörvermögen des blinden Otto; oder wenn etwa die Sequenz der traumatischen Erinnerung der explodierenden Tankstelle sich anfühlt, als wäre man inmitten eines Gewitters - mit all den sich überlagernden Donnern, den Blitzen und Wasserergüssen.

In der zeitlichen Entfaltung entwickelt sich so im Filme-Sehen von DER KRIEGER UND DIE KAISERIN aus der filmimmanenten Logik heraus ein verkörpertes Verstehen von Liebe als der Schwierigkeit, sich zu berühren, ohne sich $\mathrm{zu}$ verletzen.

Und zugleich, und damit verwoben, entsteht das Begreifen des Folgens seiner Bestimmung als Veränderung - wobei sich diese Bestimmung in der Spannung eines eigenen Willens und einer schicksalhaften Fügung bewegt.

So gibt es immer wieder Variationen und unterschiedlichste Kontexte von Bestimmungen, Plänen, Planungen und Willensbekundungen, die sich in zweierlei Hinsicht auf Zustandsänderungen beziehen: Vor allem um Abstandnehmen geht es dabei, genauso wie um Zusammensein. Der Film beginnt am Meer, mit der Adressierung eines Briefes, der für Sissi bestimmt ist (s. u.). Es gibt papierene Gebäudepläne, aufgrund derer es Walters Plan eines Bankraubs gibt, um den eigentlichen Plan - mit Bodo nach Australien auswandern - realisieren zu können, wobei Walters Frage an Bodo, „Wollen wir nun, oder wollen wir nicht?“, unbeantwortet im Raum stehen bleibt. Es gibt Pläne, die erst einmal nur partiell in die Tat umgesetzt wurden: Wenn Sissi mit ihrer Freundin Meike (Natja Brunckhorst), von der der Brief ist, „immer ans Meer“ wollte, bisher aber nur Meike dies „geschafft“ hat; wenn Sissi „sofort hingehen wollte“ zur Bank, um dort für Meike ein Erbstück ihrer verstorbenen Mutter abzuholen, dann aber auf dem Weg dorthin von einem Schwerlaster überfahren wird und nach ihrer Rettung durch Bodo erst einmal für Wochen im Krankenhaus liegt. Es gibt einen unbewussten Plan, der als der große, das Erleiden und Leiden beendende, Lebenszustandsverändernde Schicksalsplan empfunden wird, dem zufolge es für Sissi „was bedeutet“, dass Bodo unter dem Lastwagen war und ihr mit einem Luftröhrenschnitt Luft zum Atmen verschafft hat: nämlich dass sich ihr „Leben ändern muss“, damit nicht mehr „alles so sein wird wie vorher“ (Bodo wartet hingegen darauf, dass alles „wie vorher“ ist: wie vor der Tankstellenexplosion). „Nichts ist egal“ bei einem solchen den „Zufall“ ausschließenden Plan - so etwa, dass es Meikes Mutter ist, die von Bodo beerdigt wurde und wegen der Sissi zweimal zur Bank ging und dabei zweimal ohne eigene Absicht mit Bodo zusammentrifft: erst 
beim Unfall (den Bodo unwillentlich mitverursacht), dann beim Bankraub. Und auch ist es „nicht der Plan“, dass Bodo bei dem missglückenden Bankraub erschossen wird, denn man ist füreinander bestimmt, man ist miteinander verbunden. Es gibt des Weiteren abwehrende Aufforderungen zur bewussten Willensbekundung: mit Bodos wiederholt hingeschleuderter, an Sissi gerichteter Frage „Was willst du?“, deren Antworten („dich wiedersehen“, „wissen, ob es etwas zu bedeuten hat") immer mit harscher Zurückweisung begegnet wird. Und es gibt eine zuletzt einladende Aufforderung zur Willensbekundung:

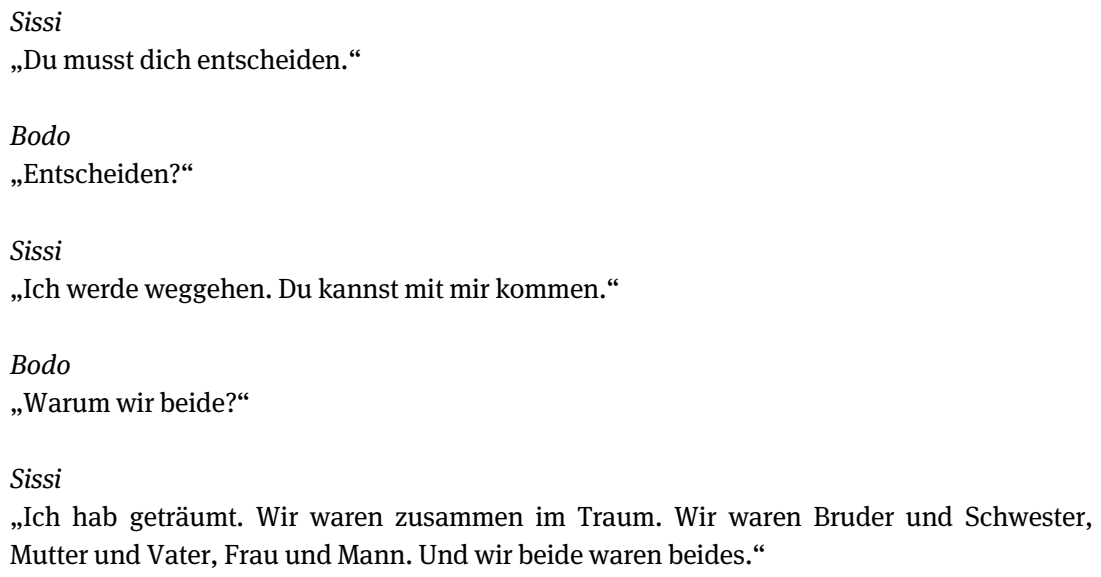

Mit jeweils selbstbestimmten Entscheidungen mündet der Film schließlich in Veränderung: im Zusammensein von Bodo und Sissi (das An-die-Hand-Nehmen), im Abstandnehmen von den bisherigen Lebenszuständen (der gemeinsame Sprung vom Dach der Psychiatrieklinik, die von der Klinikgesellschaft trennende Flucht, das buchstäbliche Verabschieden und Trennen Bodos von seinem traumatisierten, weinenden Alter Ego), und der gemeinsamen Ankunft bei Meike am Meer - und damit im Bild des Anfangs. Jene schlussendliche Veränderung versinnlicht sich im Wahrnehmungserleben des Zuschauers musikalisch, ist die gelassen-heitere Musik des Schlusses doch gänzlich anders als ansonsten im Film.

Dies fügt sich zu einem großen dynamischen Ganzen. Grundlegender Teil dessen ist ein sich wiederholendes expressives Muster, das man als Zuschauer in seiner Wahrnehmung realisiert und das eine Gegenbewegung $\mathrm{zu}$ der oben beschriebenen soghaften Ausdrucksqualität der zu heißen Berührung darstellt: das Bild einer gerichteten, mit Kraft vorandrängenden, kanalisierten, intentionalen Bewegung. Diese Bewegung drängt durch das Innere einer Briefbeförderungsmaschine und überwindet die große Distanz zwischen Meike am Meer und Sissi in der Klinik und verändert dort einen Zustand (vgl. die Analyse unten) ... Diese 


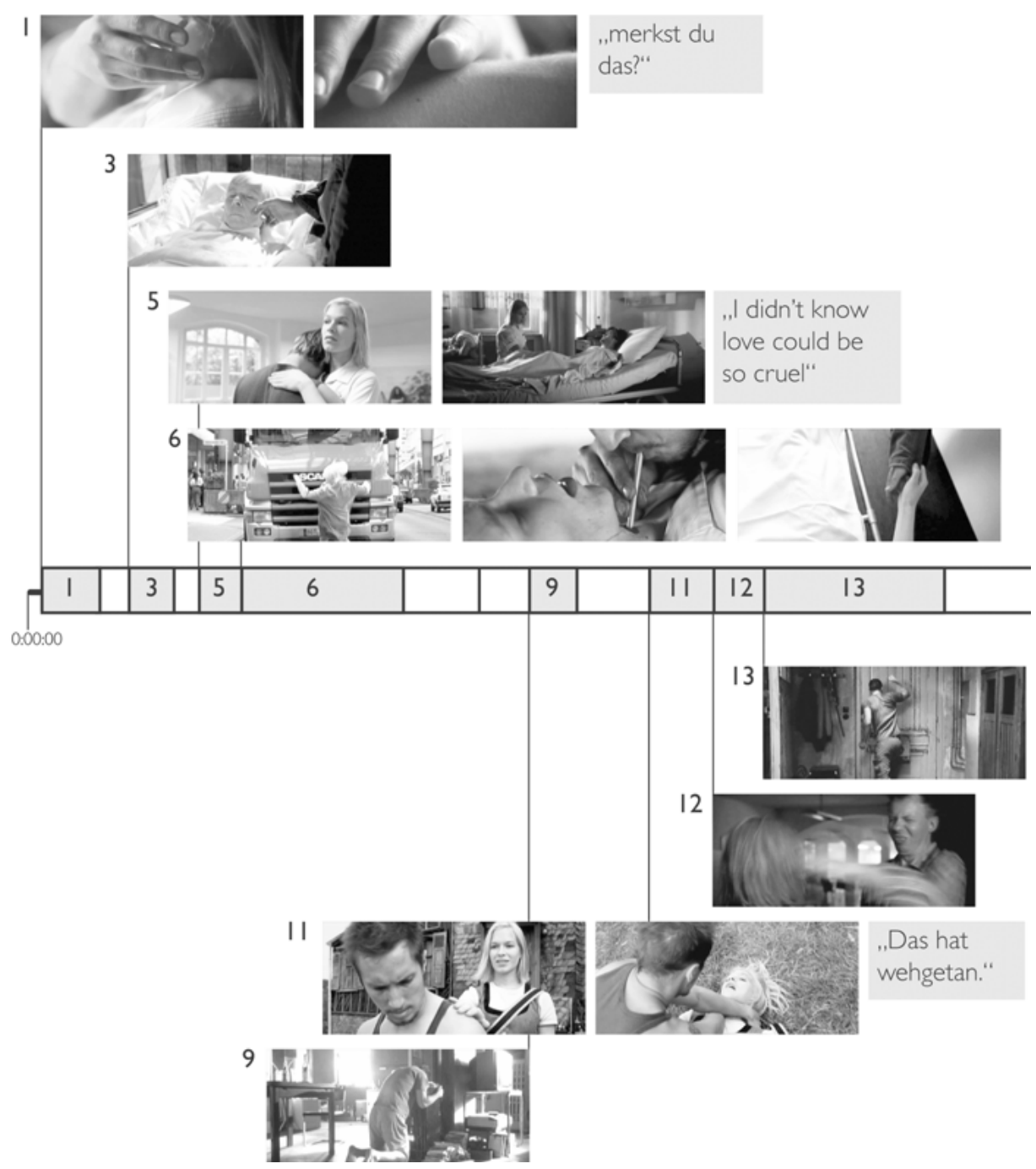

Abb. 21: Variationen von Berührungen (grau-nummeriert = dominant-relevante Szenen) in DER KRIEGER 


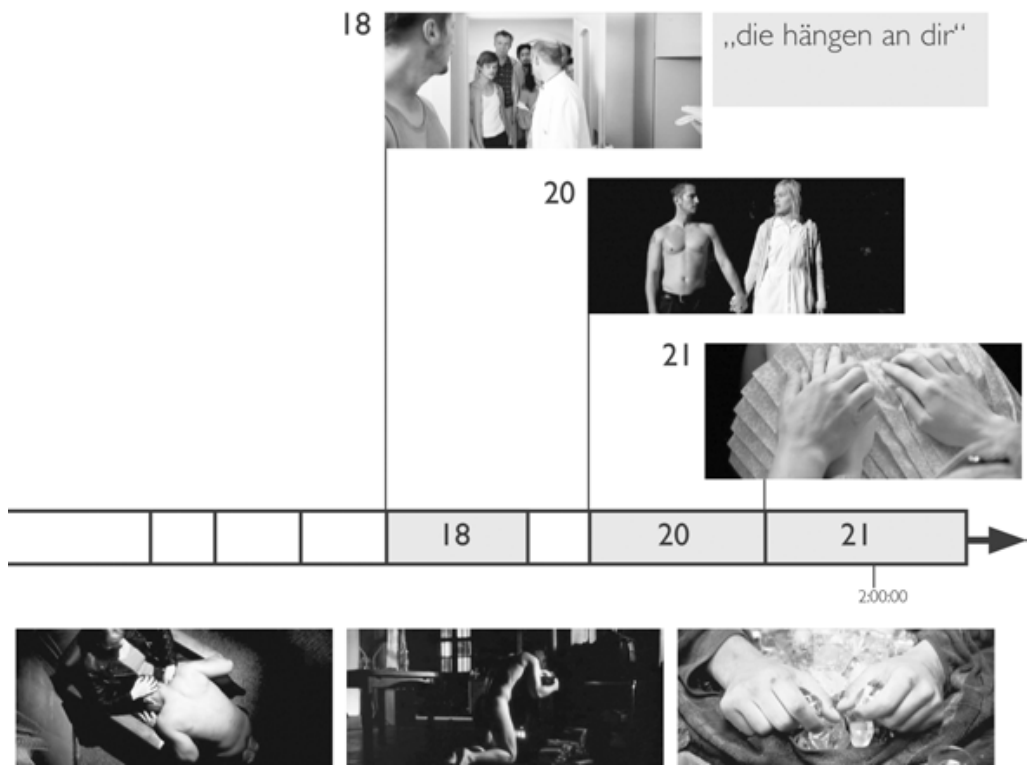

UND DIE KAISERIN. 


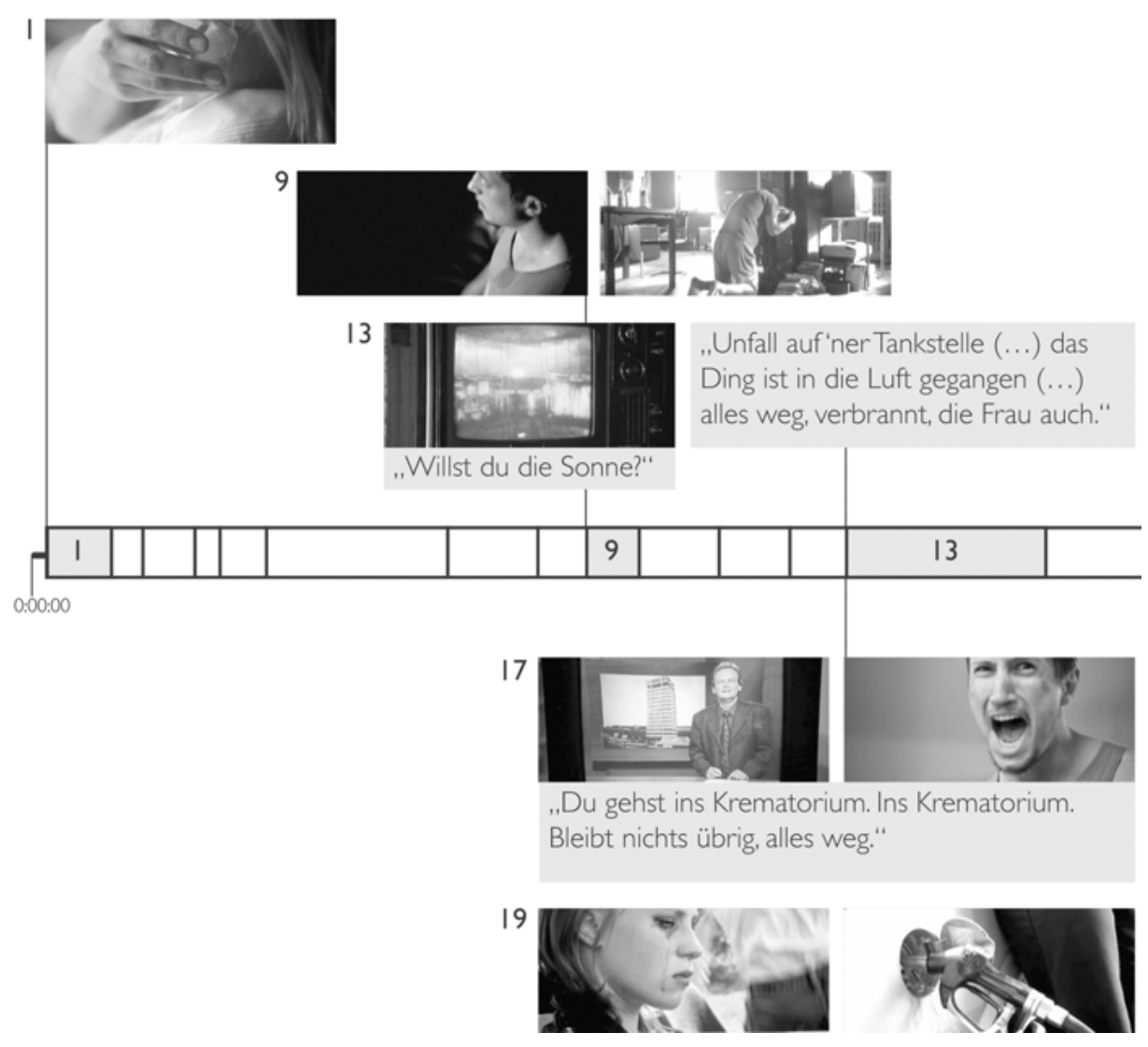

Abb. 22: Emergenz und Entwicklung des dynamischen Erfahrungsbereichs Explosion - Hitze Szenen) in DER KRIEGER UND DIE KAISERIN. 

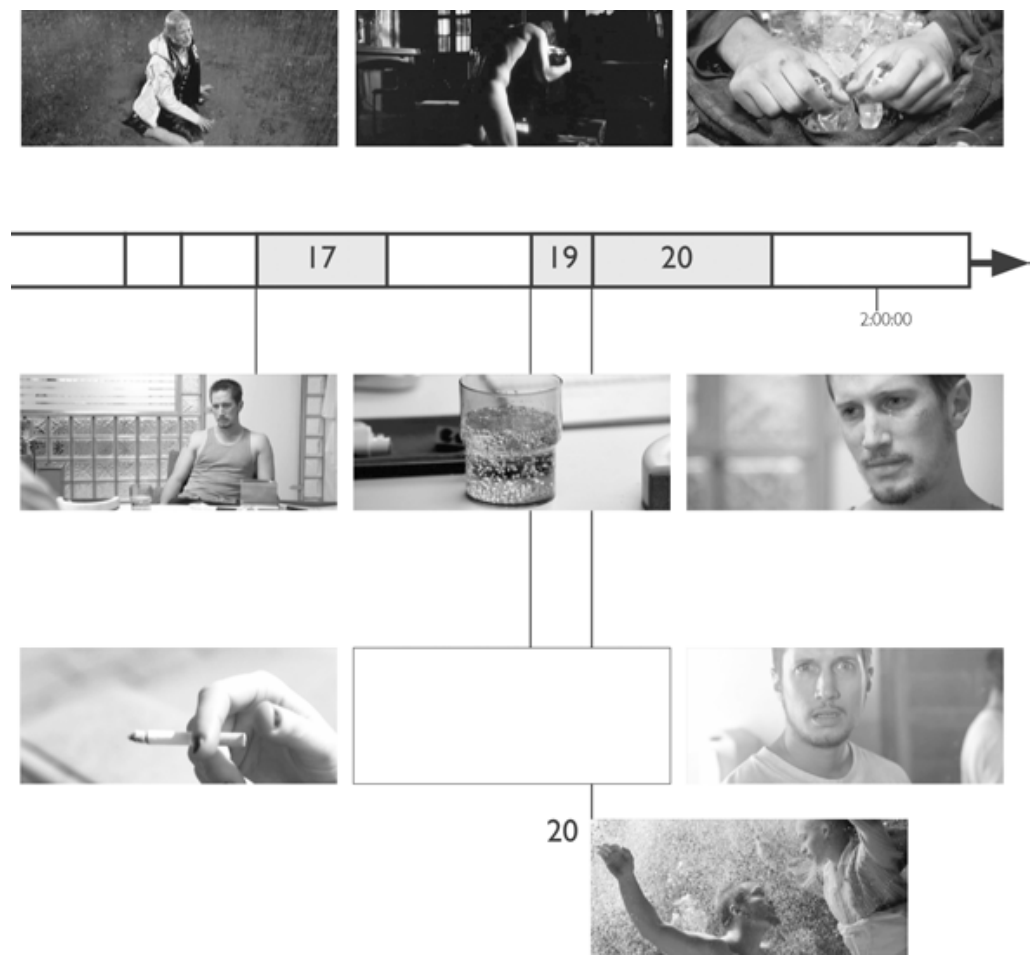

Verbrennen - Wärme - Kühlen - Eis - Wasser (grau-nummeriert = dominant-relevante 


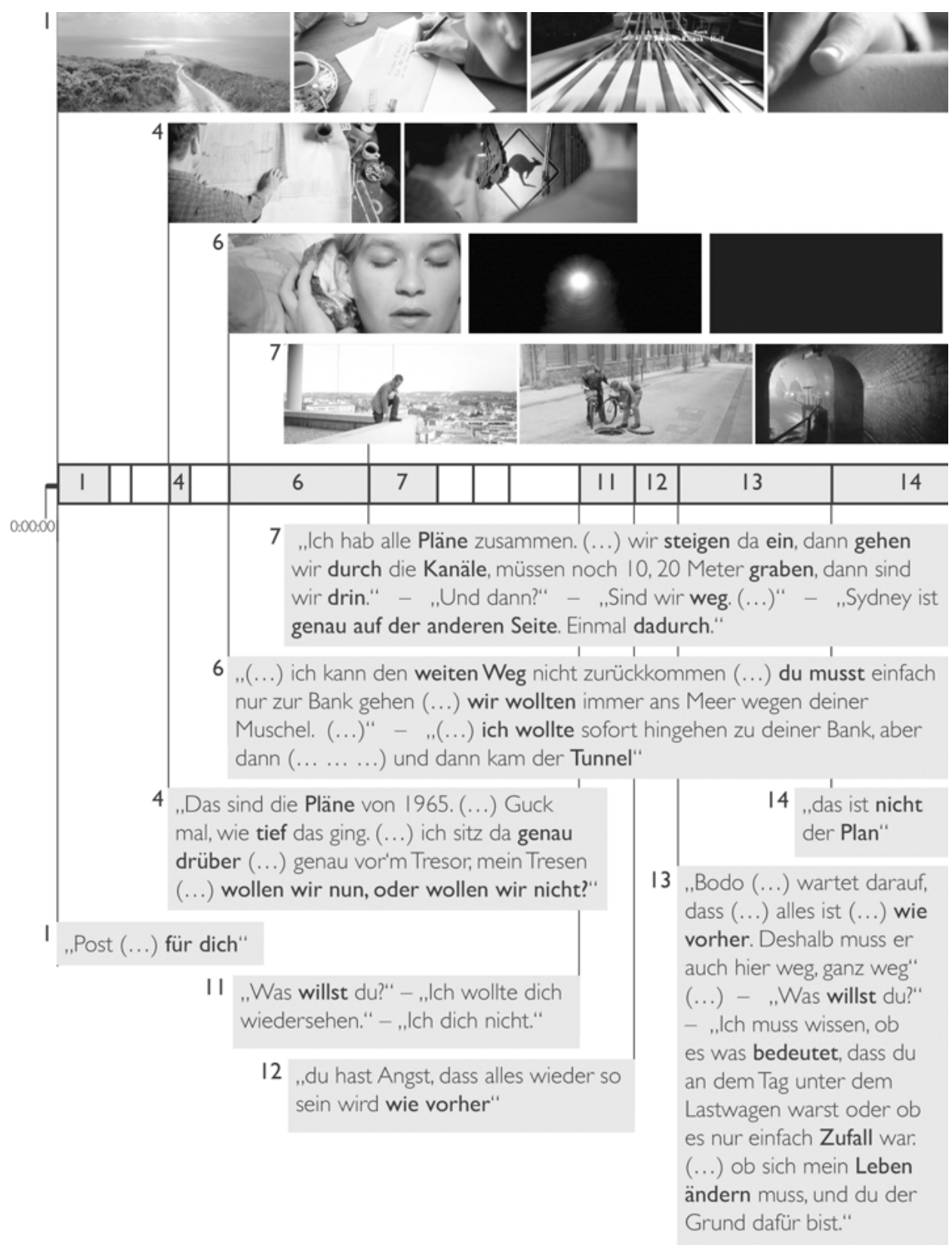

Abb. 23: Entfaltung des dynamischen Erfahrungsfeldes Tunnel - Kanal(isiert) - Verbundensein - Plan Bestimmt-Sein - Willensbekundung (grau-nummeriert = dominant-relevante Szenen) in DER KRIEGER UND 

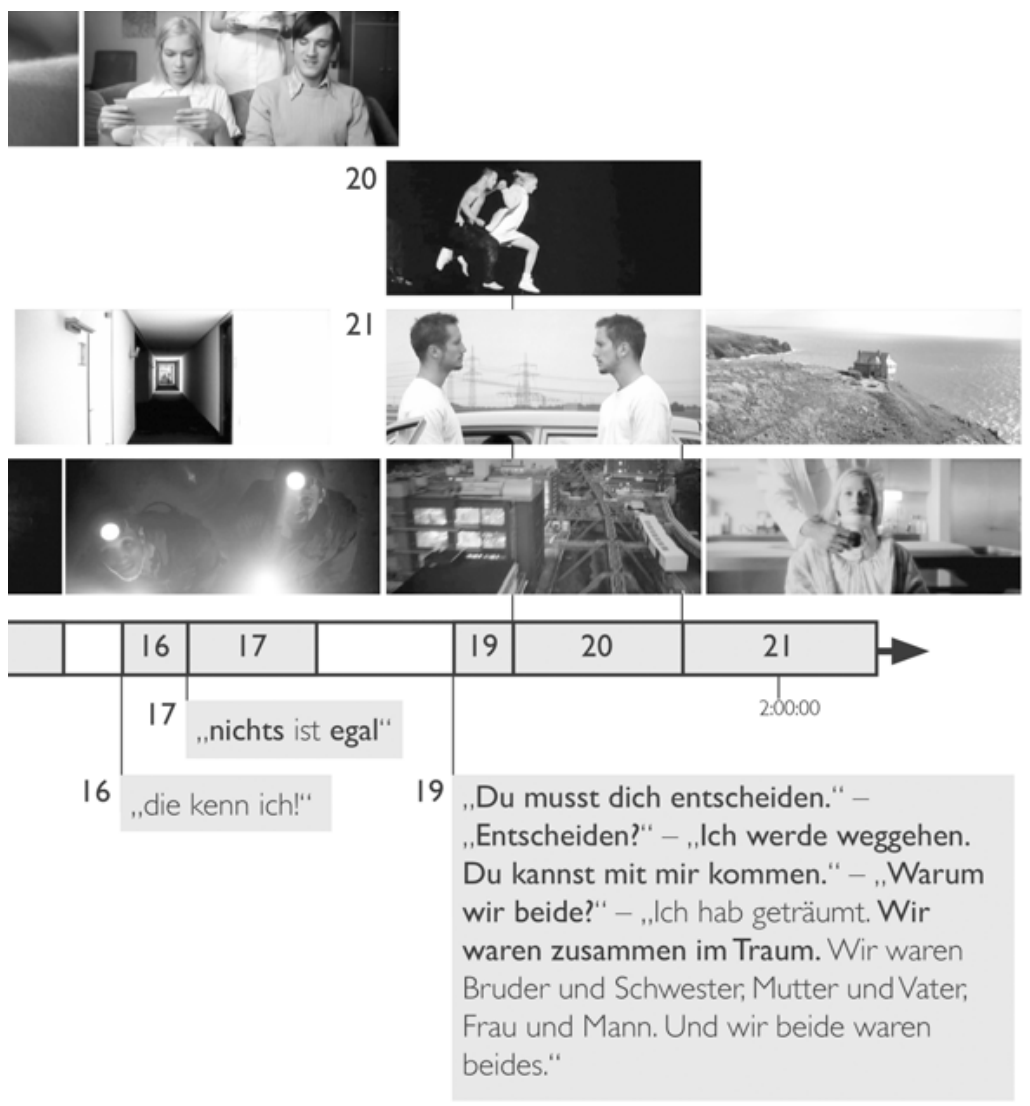

Zusammenkommen - Wegsein - Abstand gewinnen - Distanz - Veränderung DIE KAISERIN. 
Bewegung drängt schwindelerregend schnell durch einen phantastischen tiefen, langen, schwarzen, tunnelhaften Ohr-Muschel-Kanal, an dessen Ende ein gleißendes Licht kreiselnd näher und näher kommt: Alle physikalischen Raum-ZeitOrdnungen verlassend, bringt hier diese Bewegung das Gesicht Sissis - die gerade noch nachts Meikes Brief gelesen hat und sich nun mit geschlossenen Augen eine große Muschel ans Ohr hält - in der Wahrnehmung mit dem tagsüber die Straße entlangrennenden Bodo zusammen, dessen Weg sich gleich mit dem Sissis beim Unfall kreuzen wird, und wo Sissi Bodo im „Tunnel“ dann zunächst wieder verliert und nur seinen Knopf in der Hand zurückbehält ... Und diese die Gesetze von Raum und Zeit sprengende Bewegung bringt Bodo und Sissi erneut in der Wahrnehmung zusammen: wenn Walter und Bodo - die eben noch von Sidney gesprochen haben, das „genau auf der anderen Seite“ sei, „einmal dadurch“ - in ein dunkles Kanalsystem einsteigen, mit Stirnlampen Lichtkegel aus dem Dunkel dringen und ein sich anfügender fliegender Kamerablick das Gerüst der Wuppertaler Schwebebahn entlanggleitet, das sich durch die nächtliche Stadt wie eine Verlängerung des Kanalsystems schlängelt, und dies schließlich überblendet in ein Auftauchen aus einer tiefschwarzen tunnelhaften Öffnung am Hals Sissis, die im Krankenhaus gerade für ihre Entlassung fertiggemacht wird ... Und diese Bewegung bringt auch Sissis Wiederfinden von Bodo, ihren Weg zu ihm, um ihn wiederzusehen, zur Wahrnehmung.

Die vorangegangenen drei Abbildungen dienen der makroanalytischen Veranschaulichung, wie im Metaphorisieren über den gesamten Film diese beiden systematischen Metaphern, wie sie hier skizziert waren, entstehen: Abb. 21 zeigt eine Makrografik des gesamten Films mit Markierung der Szenen der Berührungsvariationen. Abb. 22 zeigt Markierungen der dominant-relevanten Szenen, in deren Zusammenhang der dynamische Erfahrungsbereich Hitze - Verbrennen - Wärme Kühlen - Eis - Wasser emergiert und sich entwickelt. Abb. 23 zeigt Markierungen der Szenen, in denen sich das dynamische Erfahrungsfeld Tunnel - Kanal(isiert) Verbundensein - Zusammenkommen - Wegsein - Abstand gewinnen - Distanz Veränderung - Bestimmt-Sein - Plan - Willensbekundung entfaltet. (Für eine tabellarische Übersicht der gesamten Szeneneinteilung mit Timecodes und einer Benennung der Szenen mit Arbeitstiteln vgl. Abb. 52 im Anhang.)

Charakteristisch für das filmische Metaphorisieren, wie es DER KRIEGER UND DIE KAISERIN modelliert, ist, dass Szenen wiederholt vielfältig in ihrer Orientierung sind, verschiedene Dimensionen bearbeiten: die Liebe und das Berühren, das Suchen und das Überwinden von Distanz, das Festhalten am oder Gefangensein im Alten sowie die bestimmte Veränderung. Es ist ein Oszillieren, weil oft mehr als nur ein Fokus in einer Szene gegeben ist (diese Überlagerungen zeigt auch ein Abgleich der Makrografiken) oder Dimensionen subdominant weiter da sind, um später wieder dominant zu werden. Sprache und die Ebene des audiovisuell 
gegenständlich Dargestellten haben an diesem Metaphorisieren genauso ihren Anteil wie die audiovisuelle Inszenierung, die all dies erst zum Ausdruck und zur Wahrnehmung bringt.

Es geht den folgenden Analysen nun nicht um eine umfassende Rekonstruktion dieses komplex-rhizomatischen ${ }^{4}$ Metaphorisierens mit seinen Verschiebungen, Überlagerungen, Verzweigungen, Elaborierungen, multiplen Verbindungen etc. - das darin noch Ähnlichkeiten zu verTigo hat. Vielmehr werden mit drei Analyseabschnitten einige ausgewählte Passagen des Metaphorisierens mikroanalytisch betrachtet, um aufzuzeigen, wie dort ein Wahrnehmen, Fühlen und Verstehen modelliert wird.

Der Auftakt sei mit dem Filmanfang gemacht, liegt doch darin auch der Beginn der filmischen Weltkonstitution. Hier beginnt $\mathrm{zu}$ emergieren, was sich dann rhizomhaft ausbreitet. Hier nimmt das Metaphorisieren seinen Anfang: mit einer Emergenz von Erfahrungen des Bestimmt-Seins und Distanz-Überwindens, von Berührung und Sensibilität und Veränderung. Die erste Szene steht somit auch beispielhaft für die oben angesprochene szenische Mehrdimensionalität. Mit den beiden weiteren Analysen liegt der Schwerpunkt dann auf einigen ausgewählten Dynamiken und Entwicklungen der Liebes-Thematik, einmal mit Blick auf die Protagonistin Sissi, und einmal mit Blick auf den Protagonisten Bodo. Ist die erste Szene noch sehr reduziert im Sprachausdruck, spielen bei den dann zu betrachtenden Szenen bzw. Szenenausschnitten sprachliche Äußerungen teils eine dominante Rolle: Äußerungen, die für sich betrachtet zunächst einmal wenig metaphorisch scheinen, die ihre Metaphorizität jedoch auf ganz eigene Weise dadurch gewinnen, dass sie konstitutiver Bestandteil filmischer Bewegungsbilder sind.

\section{Anfangen: Bestimmt sein, Distanz überwinden, sensibel sein für Berührungen, intendierte Veränderungen}

,Post für Sissi‘: erste Szene, 0:00:14-0:05:05

Die Geschichte nimmt ihren Anfang mit dem Brief einer Frau, der für Sissi bestimmt ist. Der Ausgangspunkt dieses Briefes: ein einsames Haus am Meer in Frankreich, sein Ziel: die psychiatrische Abteilung eines Klinikums in Deutschland. Was sich in den fast fünf Filmminuten entfaltet, die zwischen diesen beiden Punkten liegen, ist im szenischen Ganzen (dominant durch die Musik gestaltet, und drei Ausdrucksbewegungen zueinander fügend), das Bild einer anschwellen-

4 Zum Rhizom vgl. Gilles Deleuze/Félix Guattari: Rhizom [1976]. Berlin: Merve 1977. 
den Kraft, welche zuletzt in einen in sich ruhenden, auf die Gegenwart konzentrierten Zustand vordringt und diesen verändert. Mit die Wahrnehmung ästhetisch organisierenden Inszenierungen von Erfahrungen des Bestimmt-Seins und des Überwindens von Distanz, des Berührens und der Sensibilität sowie der intendierten Veränderung beginnen hier für das weitere Metaphorisieren zentrale Linien. Dieses Entstehen sei nun aufgezeigt.

Mit einem leisen, hohen Klang im schwarzen Bild beginnt alles mit einer reduzierten Flächigkeit. Doch dann weitet sich in der Eröffnung des Films (erste Szene, erste Ausdrucksbewegungseinheit: 0:00:14-0:03:16, Abb. 24) die Wahrnehmung auch schon auf eine tiefe Räumlichkeit hin: mit einem zentralperspektivischen Panoramablick auf eine üppige Landschaft am Meer, durch die sich ein Feldweg zu einem Haus ganz vorne an der Küste schlängelt. Wind rauscht. Möwen schreien. Dieser so geweitete filmische Raum, in dem in diesen allerersten Minuten zur Wahrnehmung kommt, wie eine Frau einen Brief adressiert und einwirft und wie der weitere Weg dieses Briefes verläuft, füllt sich, ganz allmählich und Schicht um Schicht, mit einem immer komplexer gestalteten dynamischen Muster. Ein sich intensivierendes Vorantreiben und -drängen wird sich hier entfalten mittels sukzessiv erklingender, ineinandergreifender audiovisueller Rhythmen und Tempi, wie im Folgenden rekonstruiert sei.

Mit dem Einstellungswechsel vom Panorama hin zur Nahaufnahme einer Frau, die einen Brief adressiert, ersetzen ein schnelles gleichmäßiges Weckerticken, das schnelle Kratzen einer Schreibfeder und das Rascheln der Briefpapiere das Meeresrauschen. Diese diegetischen Sounds werden überlagert und schließlich abgelöst von einem ebenso schnellen und rhythmischen Anschlagen eines hohen Glockentons. In der Folge wird der Sound peu à peu immer dichter und treibender, in dem immer mehr Instrumente hinzukommen: Ein pulsierendes und unablässig fließendes Wechseln zwischen Streichertönen setzt ein, es kommt mit tiefen Klaviertönen der stetige Grundschlag eines 4/4-Takts hinzu, während die Frau das Haus verlassen hat und mit zügigen Schritten den langen Weg hinaufgeht, um den Brief schließlich in einen Briefkasten zu werfen.

Dieser Moment ist ein Kulminationspunkt: Die pulsierende Musik dauert fort, doch zugleich ist nun alles gedehnt und verlangsamt. In extremer Untersicht ist die dunkle Innenansicht eines Briefkastens gegeben. Durch den Schlitz fliegt der Brief in Zeitlupe mit einem dumpfen Rauschen in weitem Bogen durch den dunklen Bildraum. Die darauffolgende Einstellung verlängert diese Bewegung des Eintauchens noch: Während der Brief, immer noch in Zeitlupe, weich auf anderen Briefen landet, zoomt die Kamera langsam, aber kontinuierlich an den Brief heran, bevor das Bild ins Schwarz blendet. Und noch tiefer taucht man ein, wenn aus dem Schwarzbild der weiße Filmtitel-Schriftzug aufblendet, subtil noch größer wird und das Bild erneut im Schwarz versinkt. 
Eine beschleunigte Montagesequenz statischer, jedoch in sich selbst hochdynamischer Einstellungen schließt sich an: Verschiedenste Bildachsen - Diagonalen, Horizontalen, Fluchtpunktperspektiven -, rund und kantig geformte visuelle Muster und immer wieder wechselnde Bewegungsrichtungen und Geräuschrhythmen realisieren zusammen mit dem noch treibender gewordenen Sound der Musik einen höchst komplexen und nicht wirklich vorhersehbaren und dennoch einer immanenten Ordnung folgenden, zielstrebigen und unaufhaltsamen Weg des Briefes durch das mechanische Innenleben einer Briefbeförderungsmaschine. Zuletzt rutschen Briefe in gelben Verteilkisten, und eine lange, gleitende Kamerabewegung folgt einem schnell radelnden Briefträger auf einem geschwungenen Weg zum Eingang des Klinikums, bis dieses in seiner Architektur schlosshafte Gebäude in einer Totalen imposant das gesamte Bildfeld ausfüllt.

Jetzt, wo der für Sissi bestimmte Brief bereits den ganzen weiten Weg vom Meer zurückgelegt hat und an der Pforte übergeben worden ist - wo er eintauchte in den Briefkasten und durch die Tiefen und komplexen Windungen der Maschine unvorhersehbar und doch unaufhaltsam und zielgerichtet vorandrängte - jetzt, nach dieser hochenergetischen komplexen audiovisuellen Bewegung, die eine große Distanz überwunden hat, hebt eine neue Ausdrucksqualität an (erste Szene, zweite Ausdrucksbewegungseinheit: 0:03:16-0:04:14, Abb. 25). Auf der Soundebene klingt die vorantreibende Energie aus, und alsbald ist das erste Mal eine Stimme zu hören. Sie spricht sanft und ruhig. Und auch nur zwei Sätze.

\section{Frauenstimme}

„Merkst du das? Das ist Gänsehaut.“

Alles Pulsieren, alle Rhythmisierungen sind zwischenzeitlich aus dem Sound verschwunden. Nur noch der hohe, sanfte Legatoklang ist da sowie leises Atmen. In diese Ruhe erklingt reduziert und langsam die Melodie des Leitmotivs mit einer Zimbel. Und noch jeder filigran angeschlagene Ton konturiert sich so klar spürbar vor der Legatofläche wie die aufgestellten Härchen einer erschauerten Haut.

$\mathrm{Zu}$ sehen ist währenddessen die Großaufnahme einer Schale, die vor einem nur fragmentarisch gezeigten Körper steht. Die Schale ist gefüllt mit Eiswürfeln und Eiswasser. Eine Hand greift hinein, fischt einen der Eiswürfel hinaus und führt diesen an den Hals. Ein Tropfen fällt herab, als der Eiswürfel die warme Haut berührt. Ein sich ausdehnender, schwebender Moment von Sinnlichkeit hat begonnen, in dem das Voranschreiten der Zeit gänzlich aussetzt. Ruhig und ganz langsam blenden Detailaufnahmen von geöffneten Augen und Lippen sowie einer Hand, die ganz sanft über die Härchen eines Unterarms streicht, mit sehr geringer Tiefenschärfe ineinander über. In der Weichheit der Bilder spürt man die Weichheit der Haut, die auch farblich das Bildfeld ausfüllt. Zwei junge Körper sind sich hier sehr, sehr nah, verschmelzen, berühren einander, ganz sanft, vertrauensvoll. 

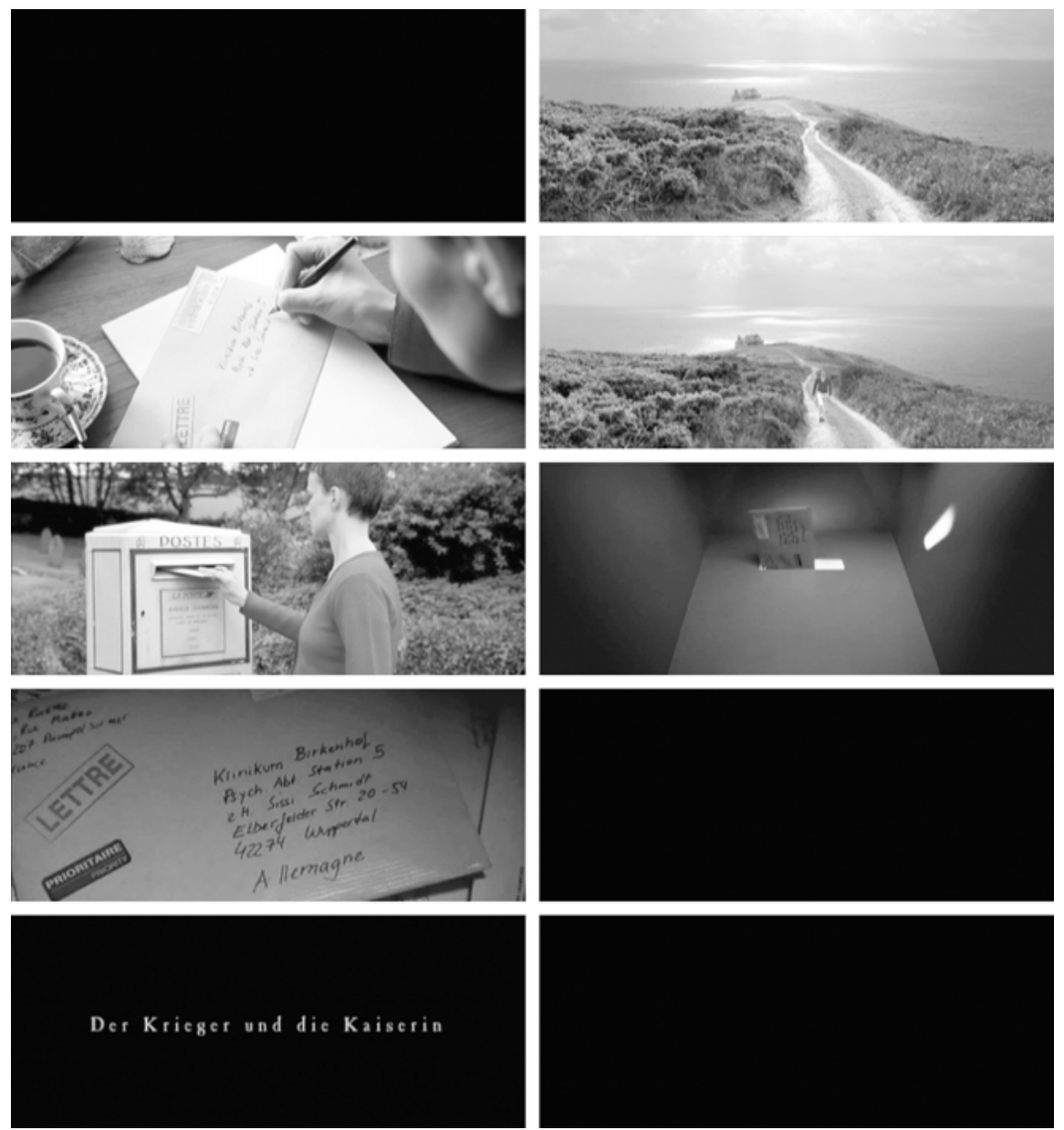

Abb. 24: Unaufhaltsames Vorandrängen, dominant gestaltet von Sound, Bildkomposition und Montage (DER KRIEGER UND DIE KAISERIN, erste Szene, erste Ausdrucksbewegungseinheit: 0:00:14-0:03:16). 

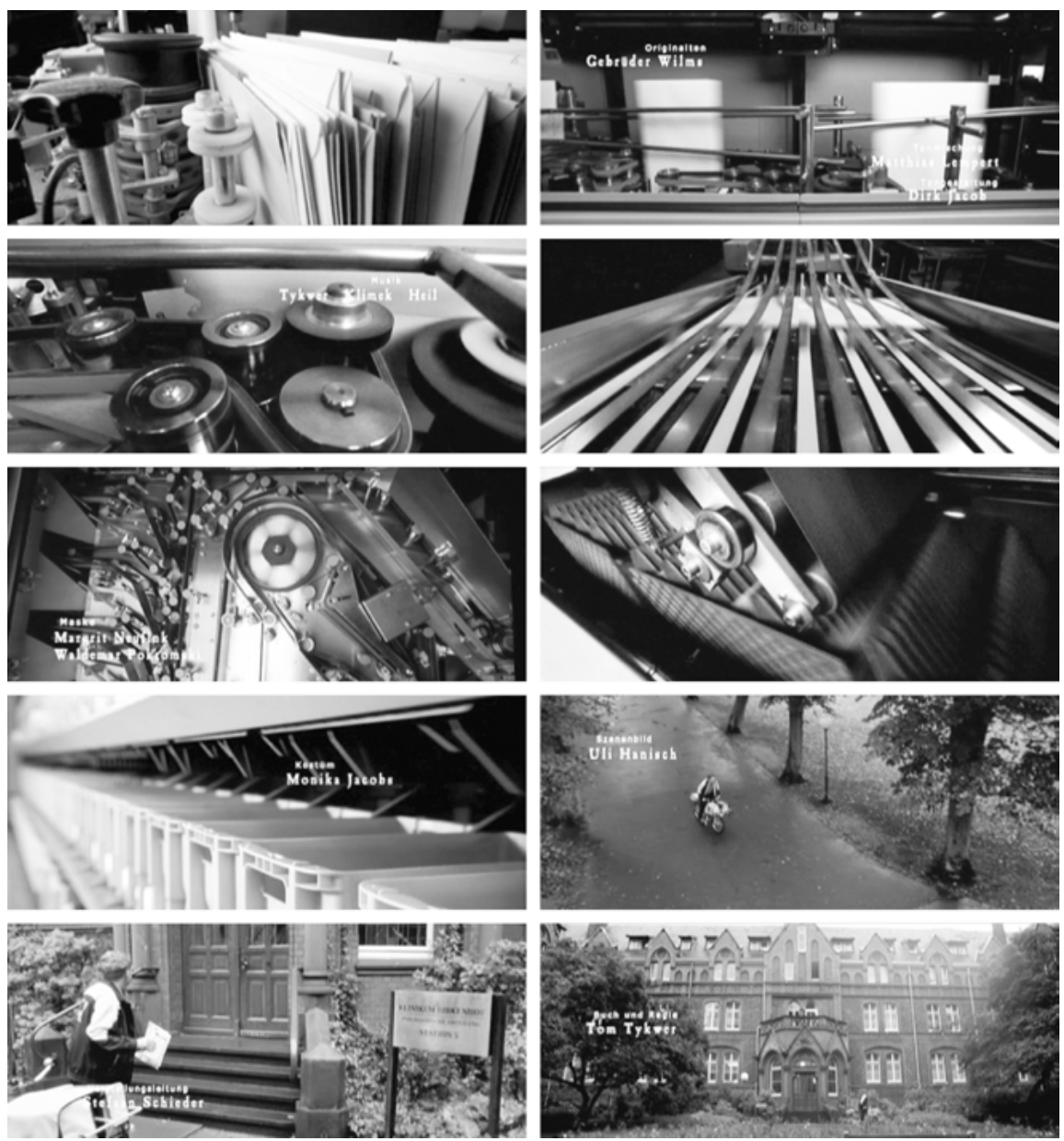

Abb. 24 (Fortsetzung) 

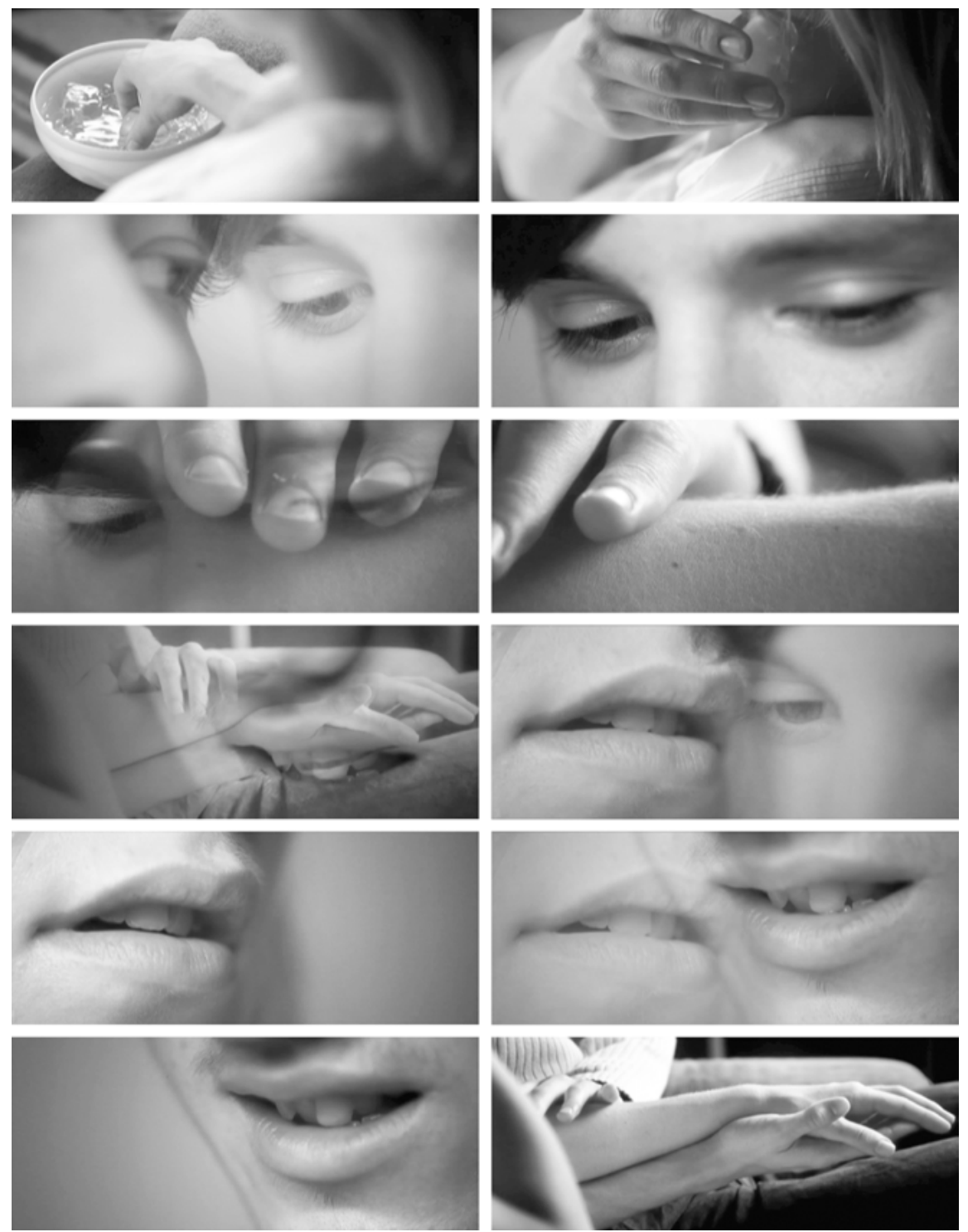

Abb. 25: In der Gegenwärtigkeit sanfter Berührung aufgehen, dominant gestaltet von Kamera und Sound (DER KRIEgER UND DIE KAISERIN, erste Szene, zweite Ausdrucksbewegungseinheit: 0:03:16-0:04:14). 

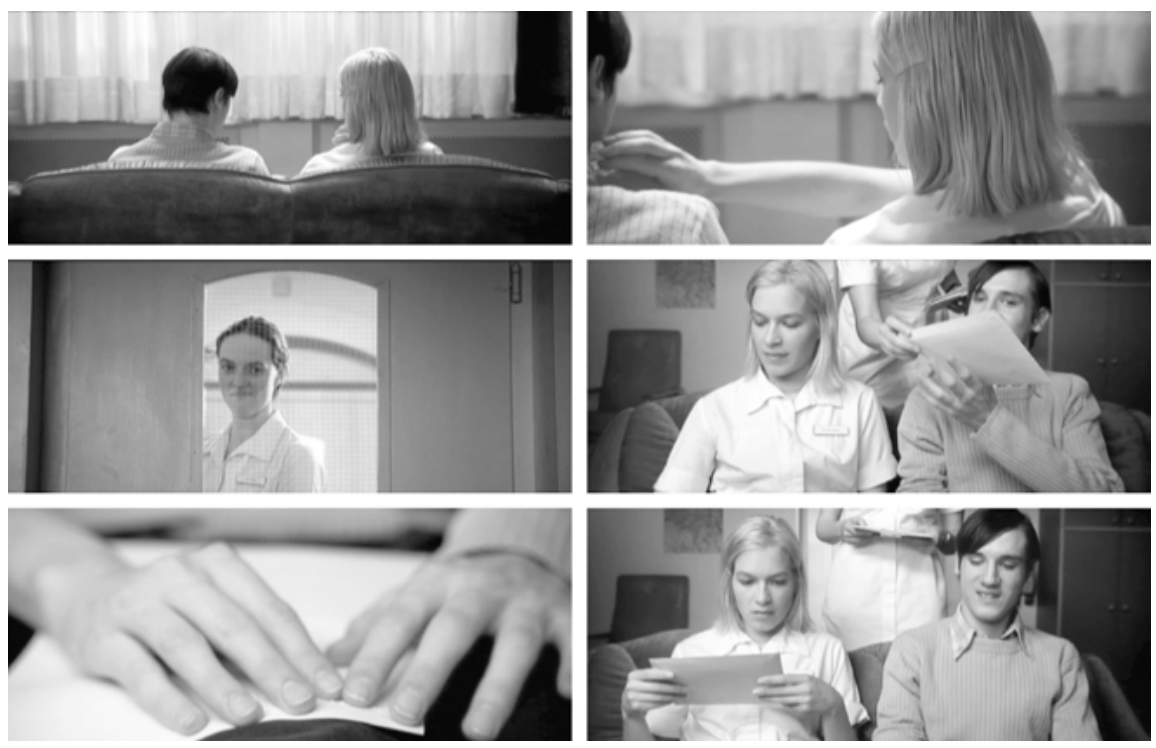

Abb. 26: Die vorandrängende Kraft dringt sanft in den Zustand der Gegenwart, des Im-MomentSeins ein und verändert diesen, dominant gestaltet von Bildkomposition, Kamera und Sound (DER KRIEGER UND DIE KAISERIN, erste Szene, dritte Ausdrucksbewegungseinheit: 0:04:14-0:05:05).

Das ganze audiovisuelle Bild ist dadurch reines Wahrnehmen, Empfinden, Spüren; ist Körper-Sein, Haut-Sein, Nah-Sein, Sensibel-Sein. Das gesamte Bild ist entspannte Intimität, ist Berührung.

Dieser Zustand verändert sich jetzt - mit Änderungen in Kadrierung, Bildkomposition, Montageform und Sound (erste Szene, dritte Ausdrucksbewegungseinheit: 0:04:14-0:05:05, Abb. 26): In der Musik setzt der vorandrängende Rhythmus sanft wieder ein, Nahaufnahmen lösen Detailaufnahmen ab, Tiefenschärfe und Symmetrien lösen Weichheit und Überlagerungen ab - und das Versunkensein in der Berührung, der Zustand reiner Gegenwärtigkeit ist Vergangenheit: durch das Eindringen der intentionalen Kraft. Mit diesem inszenatorischen Wechsel wird auch der Bildausdruck ein anderer. Das Bild reiner körperlicher Berührung transformiert sich zu einem Bild zweier separater Menschen, die mit Rollen und Namen in einer sozialen Wirklichkeit verortet sind: Die junge Krankenschwester Sissi sitzt hier neben einem jungen Patienten, dem blinden Otto, mit dem sie befreundet ist, auf einem Sofa in einem geschützten Raum, mit zugezogenen Gardinen. Mit einer weiteren Krankenschwester, die lächelnd durch die Glasscheibe einer verschlossenen Tür auf die beiden geblickt hat, bevor sie ruhig den Raum betritt, erreicht der Brief sein vorherbestimmtes Ziel: 


\section{Krankenschwester}

„Hey Sissi, da bist du ja. Ich hab hier Post für Otto. Und für dich auch.“

Sissi nimmt den für sie bestimmten Brief entgegen, hält ihn erstaunt in ihren Händen. Währenddessen berühren Ottos Hände schon längst das Papier seines Briefes und ertasten mit der Brailleschrift nun dessen Erhebungen: Berühren und Spüren, eben noch als reiner Wahrnehmungszustand gegeben, stellen sich in ihrer Bedeutsamkeit aus.

Und mit dem Verklingen der Musik ist mit diesen Wahrnehmungserfahrungen der Anfang des Films, der Anfang des Metaphorisierens gemacht, aus dessen weiterem Verlauf die beiden oben skizzierten systematischen Metaphern von Liebe als der Schwierigkeit, sich zu berühren, ohne sich zu verletzen, und dem Folgen seiner Bestimmung als Veränderung hervorgehen werden. Dem Entstehen von Erstgenannter sei sich im Folgenden gewidmet.

\section{Entwicklungen I: Ein uneingelöster Wunsch nach Distanz} ,Alltagsroutinen': fünfte Szene, 0:11:26-0:14:40

Mit der fünften Szene ist nun eine weitere Szene aus der Anfangsphase des Films betrachtet, in deren Zentrum die Darstellung sich berührender Körper steht: zunächst im Tanzen und dann bei einer sexuellen Befriedigung. Im Zuschauen bildet sich dadurch die Figur der Protagonistin Sissi weiter aus. Und hierfür werden die Erfahrungsbereiche des Berührens und der Liebe elaboriert. Das drängende Überwinden von Distanz und das Aufgehen in Berührung, das in der ersten Szene zentrale Erfahrungen waren, verkehren sich jetzt in ein nicht einzulösendes Suchen nach Distanz.

Ein immer schon doppeltes Sehen ereignet sich hier in dieser Szene. Durchgängig sind zwei Kontexte präsent. Das eine audiovisuelle Wahrnehmungsszenario, das sich entfaltet, ist das eines Tanzes zwischen einem lüsternen, etwas draufgängerischen Typen und seinem unschuldig-gefügigen Mädchen und der daran anschließenden Nacht - hierbei ist die Sprache dominant beteiligt. Synchron damit entfaltet sich das Wahrnehmungsszenario von alltäglichen Routinen in einer geschlossenen psychiatrischen Anstalt. Jedes Szenario ist im jeweils anderen zu sehen. Das dynamische Muster der Szene fügt sich aus zwei Ausdrucksbewegungen: Zunächst ist alles hell, eine fließende Bewegung, der jedoch zugleich und konstant Statik innewohnt (Musik, Kamerabewegung und Schauspiel sind hier die dominanten Inszenierungsmittel). Die darüber evozierte, sich nur vorübergehend entladende Bildspannung einer unaufgelösten Ambivalenz intensiviert sich im weiteren Verlauf, wenn alles dunkel wird, 
gepresst, eng und starr (dominant inszeniert von Montage, Schauspiel, Bildkomposition).

Die Szene beginnt mit einem Tanz (fünfte Szene, erste Ausdrucksbewegungseinheit: 0:11:27-0:13:30, Abb.27). In Großaufnahme ist ein Plattenspieler zu sehen. Der Patient Steini - er ist einer von zwei Patienten, die in einer vorherigen Szene bereits Ansprüche auf Sissi angemeldet hatten, als es aus therapeutischer Sicht darum ging, spazieren zu gehen - legt eine Schallplatte auf, und im offenen Gemeinschaftsraum der Psychiatriestation erklingt der melancholisch-romantische Evergreen „So Sorry“ aus den 1960er Jahren:

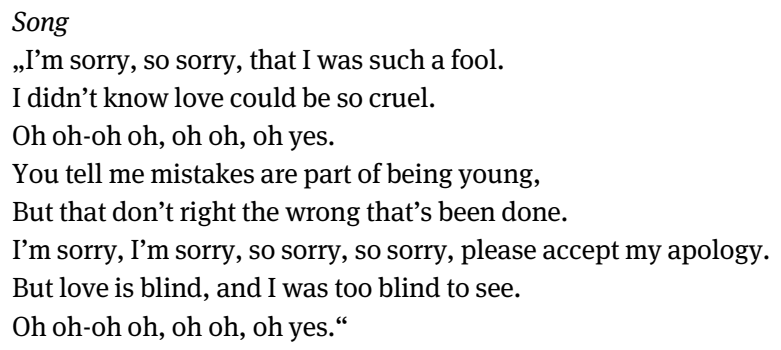

Und während Brenda Lees weicher Gesang, die hohen schmelzenden Geigen und ein säuselnder Background-Frauenchor von der unerwarteten Grausamkeit der Liebe singen, vom Falschen, das nicht wiedergutzumachen ist, und eine Entschuldigung beschwören, fokussiert der Patient Steini lüstern und draufgängerisch mit einer (nicht brennenden) Zigarette im Mundwinkel und offenem Hosenschlitz die Krankenschwester Sissi. Diese hat eben noch mit einer Kollegin gesprochen hat, jetzt aber steht sie ganz allein auf dem weiten Flur der Station, unbeholfen, unschuldig, schüchtern lächelnd. So dynamisch die Schauspielweise von Lars Rudolph hier ist, wenn er sich im Takt der Musik wiegend seines Jacketts entledigt und sich das Objekt der Begierde schließlich mit einem kehligen leisen Lachen greift, so passiv, stumm und teilnahmslos ist das Schauspiel von Franka Potente, mit ihren hängenden Armen, die von Lars Rudolph in die Höhe gerissen werden, wenn er sie zum Tanz an sich zieht, und mit ihrem ausdruckslos-distanziert werdenden Gesicht.

Ausgehend von Sissis Gesicht und Körperhaltung breitet sich die Qualität der Unbeweglichkeit, Leblosigkeit und Leere durch die anderen Gesichter und Körper, die im weiteren Verlauf dieses Tanzens im hell erstrahlenden Licht zu sehen sind, im Bild aus. Apathisch sind die Gesichter und Körper der sich im Tanz berührenden Patienten ebenso wie die der Patienten, die allein am Rand verbleiben, gefangen in ihren körperlichen Tics des Händereibens oder Fingerklopfens. Und zugleich breitet sich das Fließende und Weiche der Musik im Bild aus: in den Drehungen der Kamera, die die sich ihrerseits drehenden Paare umkreist. 

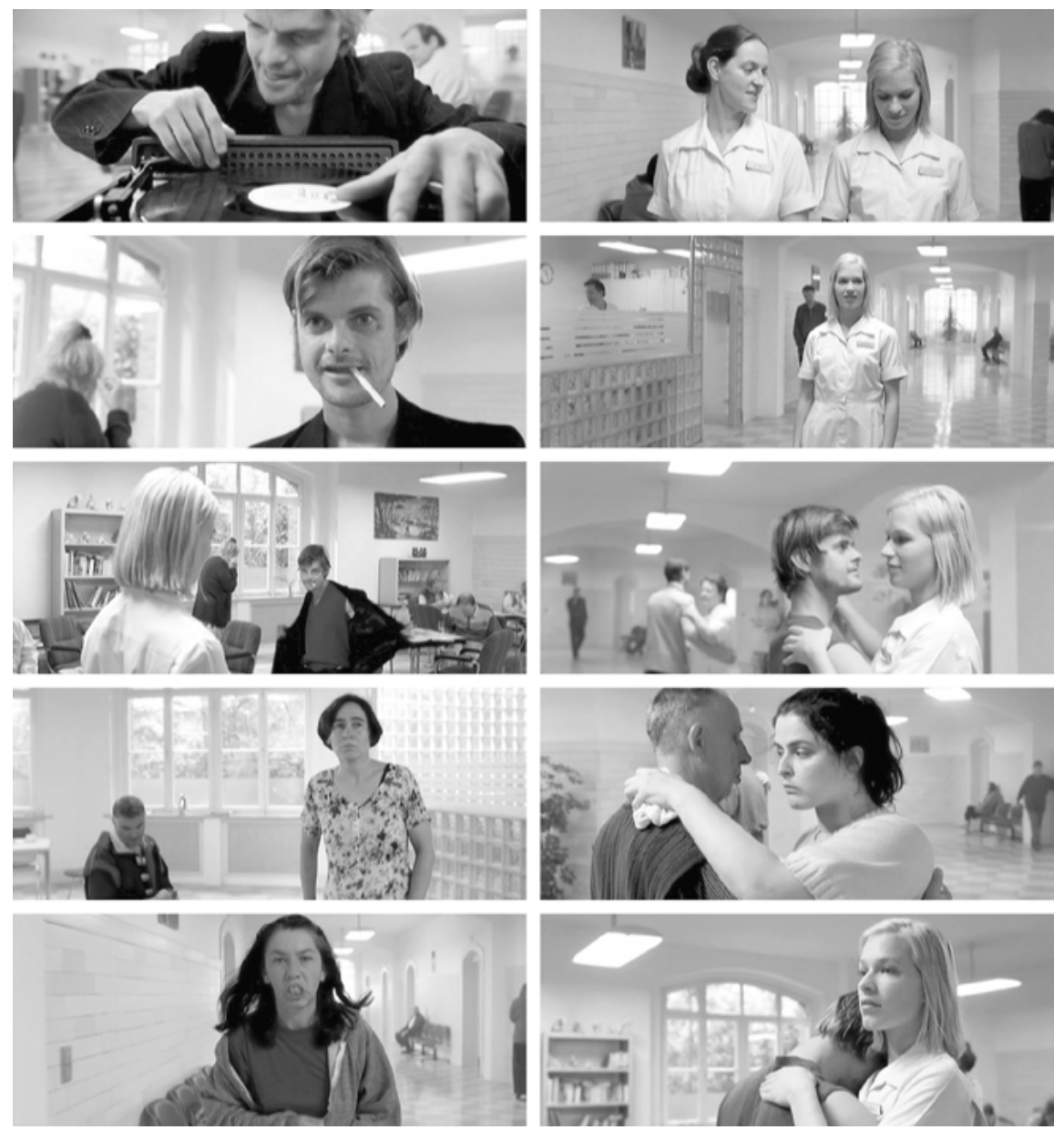

Abb. 27: Unter Spannung: Eine fließende Bewegung ist konstant verflochten mit Statik, dominant gestaltet von Musik, Kamerabewegung und Schauspiel (DER KRIEGER UND DIE KAISERIN, fünfte Szene, erste Ausdrucksbewegungseinheit: 0:11:27-0:13:30).

Die Spannung dieser unauflösbaren Ambivalenz entlädt sich für einen Moment abrupt, wenn eine Tourette-Patientin aggressiv und frontal mit einer langen geraden Kamerabewegung den Flur entlangstürmt und vulgäre Flüche ausstößt und herausschreit -

\section{Patientin}

„Schleimscheißer! Ihr schleimt euch doch alle selber voll! Arschlöcher! Wichser! Ich wichs euch einen!“ 
- bevor das Bild sich wieder fängt: im Liebeslied und dem Blick auf Steini, dessen Kopf auf Sissis Schulter ruht, und Sissi, deren ganzer Körper etwas Mechanisches hat in der Steifheit der Bewegungen und Berührungen, und deren Blick in die Ferne gerichtet ist.

Mit dem zweiten Teil dieser Szene, dessen Handlungsraum nun ein nächtlicher Patientenschlafraum ist (fünfte Szene, zweite Ausdrucksbewegungseinheit: 0:13:30-0:14:40, Abb. 28) intensiviert sich die Spannung noch. Was eben noch hell war, ist jetzt dunkel; was eben noch bewegt war, wird jetzt starr. Was eben noch fließend war, ist jetzt druckvoll. Was eben noch laut war, ist jetzt leise. Was eben noch weit war, ist jetzt eng. Im dichten Wechsel zeigen statische Großaufnahmen die Gesichter Steinis und Sissis im Dunkeln, bevor dann mit Nahaufnahmen zweier weiterer Patienten der unmittelbare Umraum einbezogen wird; die Blicke werden leer, oder sie starren; Sissis und Steinis gepresst-flüsternde Stimmen stoßen Worte hinaus, bis irgendwann nur noch unterdrücktes Stöhnen zu hören ist.

Mit dieser Inszenierung kommt der stereotype Dialog eines Paares im Bett zur Wahrnehmung: Der Mann will Sex, die Frau jedoch nicht - bis sie schließlich doch dem Drängen nachgibt, und das, obwohl auch noch jemand anderes im Zimmer schläft.

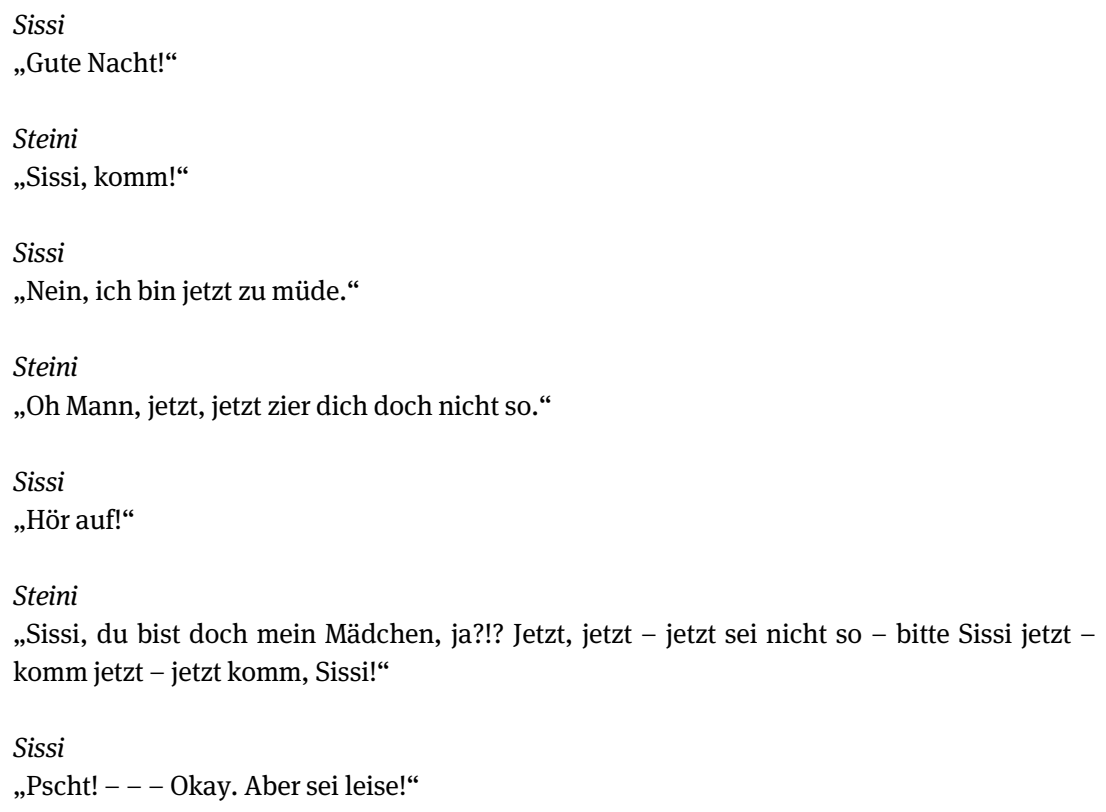

In seiner Stereotypie entwirft der Dialog für Sissi und Steini - er im Bett liegend, sie daneben stehend - das Wahrnehmungsszenario eines miteinander vertrauten 
Paares, bei dem sie sein einseitiges sexuelles Begehren notgedrungener Maßen routiniert erfüllt. Und ebendies sieht man auch, wenn Sissi - die anfänglich zu Steini noch wie zu einem Kind, das ins Bett gebracht wird, „Gute Nacht“ sagte und seine Bettdecke feststeckte - zuletzt ihre Hand unter die Decke schiebt und der Routine nachgeht, Steini damit zu befriedigen. Und dabei - ebenso routiniert - von anderen Patienten beobachtet wird.
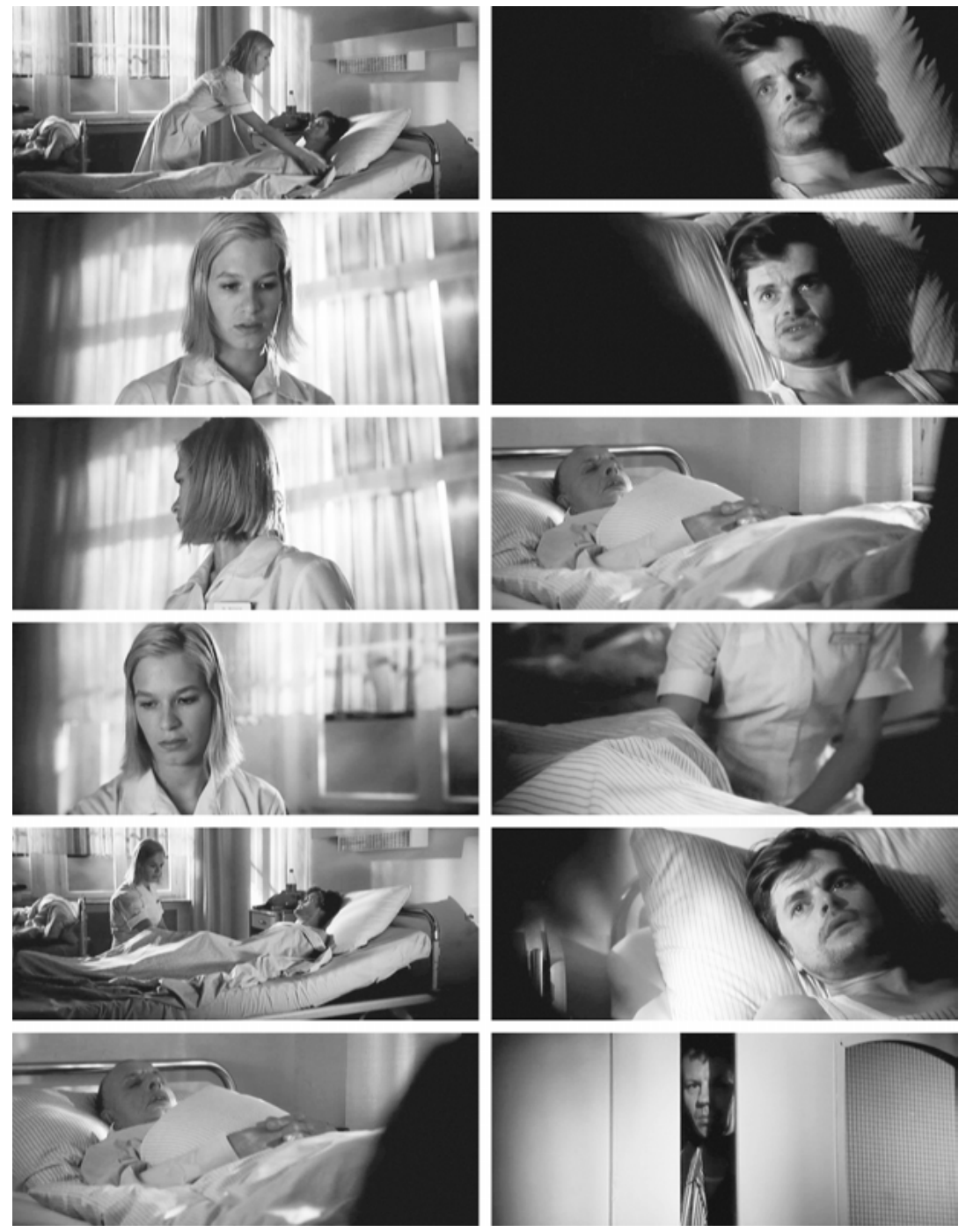

Abb. 28: Eine druckvolle Enge, dominant gestaltet von Sound, Kamera, Bildkomposition (DER KRIEGER UND DIE KAISERIN, fünfte Szene, zweite Ausdrucksbewegungseinheit: 0:13:30-0:14:40). 
Rückwirkend betont diese Handlung, dass der vorherige Ausruf ,ich wichs euch einen“ ein Element beider Wahrnehmungsszenarien ist, die sich in dieser Szene kontinuierlich miteinander verschränken: Der Satz ist Teil des Szenarios einer geschlossenen Psychiatrie, in der es Patienten mit Wahnvorstellungen und Tics gibt (ganz gleich, ob diese Tics nun das Ausstoßen obszöner Fäkalflüche sind oder die Wiederholung immer gleicher Handbewegungen), und in der es Pflegepersonal gibt, das sich um diese Patienten tags wie nachts routiniert mit körperlichem Einsatz kümmert. ${ }^{5}$ Zugleich aber ist der Satz „ich wichs euch einen“ Teil eines Wahrnehmungsszenarios von Liebe - einer Liebe, die grausam, falsch, obszön, vulgär, rücksichtslos, grenzüberschreitend, erdrückend und voller Zwänge ist, verletzend.

Diese durchgängig ko-präsenten Szenarien entfalten sich simultan und durch die sie artikulierende filmische Expressivität in einem Gefühl unaufgelöster Ambivalenz und steigender Anspannung. Mit einer distanzierten, unter ständigem Berührungszwang stehenden Krankenschwester-Patient-Beziehung und mit einer physisch intimen heterosexuellen Beziehung, in der durchgehend versucht wird, auf Abstand zu gehen, erfährt man einen uneinlösbaren, immer mehr unter Spannung stehenden Wunsch nach Distanzierung und Abstand im Erleben von Intimität und physischer Nähe. (Abb. 29)

Für die mikroanalytische Betrachtung von Prozessen filmischen Metaphorisierens als einer Zuschaueraktivität ist hier schließlich auch der fließende Wechsel von einer Szene zur nächsten interessant. Denn die unter Spannung stehende Simultaneität der Wahrnehmungsszenarien von Psychiatrie und Liebe löst sich mit dem Beginn der nächsten Szene auf. Mit Sissis Betreten des Stationszimmers, verbunden mit den Worten „So, jetzt schlafen alle“, schwindet der Liebes-Kontext aus der Darstellung, und es bleibt allein der der Psychiatrie - wobei die Routine alles Vorherigen rückwirkend noch einmal betont wird: Sissi hat ihre (Liebes-) Dienstschicht beendet und verabschiedet sich müde von ihren Kollegen, die schon die Medikationen für den nächsten Tag vorbereiten. ${ }^{6}$

5 Dass Tanzen für das übrige Pflegepersonal nicht nur selbstverständlicher, sondern auch unverfänglicher therapeutischer Klinikalltag ist, zeigt sich im entspannt-professionellen Körperausdruck jener Schauspielerin, die eine ältere, ebenfalls mit einem Patienten tanzende Pflegerin spielt.

6 Nicht zuletzt die hier besprochene fünfte Szene von DER KRIEGER UND DIE KAISERIN und der Beginn der darauffolgenden vollzieht eine Aneignung von Formans ONE FLEW OVER THE CUскоо's NEST, hier insbesondere der Anfangsszene und ihrem Medication Valse. 
Audiovisuell inszenierte Szenarios

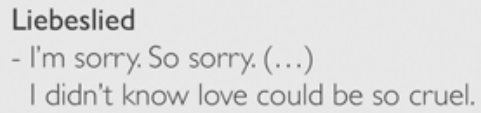

Patientin

- Wichser! (...)

Ich wichs euch einen!
Teilnahmsloses Schauspiel

Passivität

vs. dynamisches Schauspiel

Aktivität

\section{Psychiatrie}

Krankenschwester / Patient

Tanzen

Unschuldiges Mädchen / Draufgänger

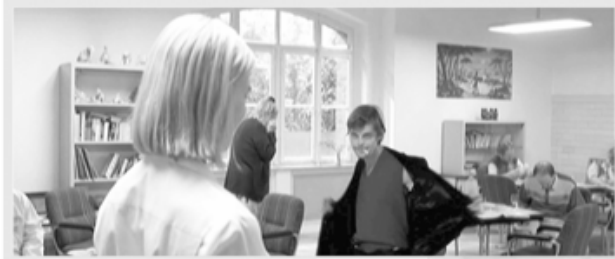

f
Psychiatrie

Patientin
Ausdrucksbewegung

Szenische Komposition fließende Bewegung (gerade - rund - gerade - rund), konstant verflochten mit Statik

spannungsvolle, unaufgelöste Ambivalenz

Abb. 29: Liebe und Psychiatrie: Simultane Entfaltung zweier aufeinander bezogener 


\section{Liebeslied}

- Mistakes are part of being young but that don't right the wrong that's been done.

\section{Teilnahmsloses Schauspiel \\ Passivität}

\section{Psychiatrie \\ Krankenschwester / Patient \\ Tanzen \\ Mädchen / Mann}

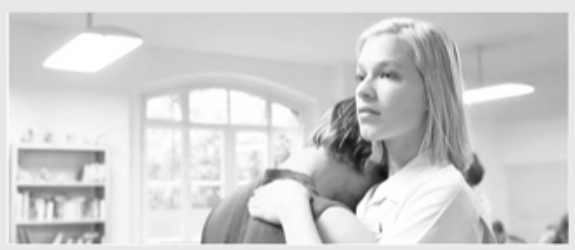

\section{Krankenschwester/Patient}

- Gute Nacht!

- Sissi, komm!

- Nein! Ich bin jetzt zu müde!

- Oh Mann, jetzt zier dich doch nicht so.

- Hör auf?

- Sissi, du bist doch mein Mädchen, ja?!? (...) jetzt sei nicht so! (...) jetzt komm!

- Pscht! - - Okay. Aber sei leise!

\section{Angespanntes Schauspiel \\ Spannung}

\section{Psychiatrie}

Nachtdienstroutine

Sex

routinierte manuelle Befriedigung unter Zwang

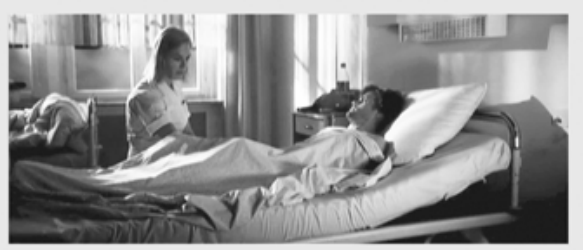

druckvolle Enge

steigende Anspannung

Wahrnehmungsszenarien für Berührungen (DER KRIEGER UND DIE KAISERIN, fünfte Szene: 0:11:26-0:14:40). 
Dass hier eine neue Szene beginnt, während zugleich die vorherige Szene noch zu ihrem Abschluss kommt, evoziert dominant die einsetzende Musik, mit der an das Ende der oben besprochenen Eröffnungsszene angeknüpft wird (bei der Sissi zuletzt den für sie bestimmten Brief in den Händen hielt, den sie jetzt - endlich lesen wird), aber auch die weitere inszenatorische Gestaltung. Ein neues Bewegungsmuster hebt hier an, und es erfolgt eine neue Ausrichtung: Jene Szene beginnt nun ihren Lauf zu nehmen, in der sich die Wege von Bodo und Sissi zum ersten Mal beim fast tödlichen Autounfall auf brutal-gewalttätige Weise kreuzen, und man in diesem Autounfall Sissis Sich-Verlieben in Bodo erfährt. ${ }^{7}$

\section{Entwicklungen II: Der Sog der zu heißen Berührung}

Um das Entstehen des Verständnisses von Liebe als der Schwierigkeit, sich zu berühren, ohne sich zu verletzen, wie es über den gesamten Film emergiert, exemplarisch zu rekonstruieren, wurde im Vorangegangenen eine Szene betrachtet und wie dort mit Blick auf die Figur Sissi die Erfahrungsbereiche Liebe und Berührung entwickelt wurden. Im Folgenden wird ein Element einer Linie der Liebe und des Berührens rekonstruiert (und damit aus den Verzweigungen herauspräpariert), mit der im Metaphorisieren vor allem die Figur von Bodo im Zuschauen entsteht, aber auch Sissis Verbundenheit mit ihm. Die Ausdrucksqualität eines langsam-gedehnt Soghaften, das zuletzt konterkariert wird, ist hierbei prägend. Zum Ausdruck gebracht wird damit ein Wahrnehmungserleben $\mathrm{zu}$ heißer Berührung. Ein solcher Sog ist wiederholt inszeniert, und im Metaphorisieren bilden hier mehrere Ausdrucksbewegungen eine Verbindungslinie, auch über Szenengrenzen hinweg. Die Analyse zeigt, wie über und in Variationen und Wiederholungen ein Denken an einen geliebten Menschen, wie Sehnsucht als eine den physischen Körper bedrohende Sogerfahrung einer Berührung, die zu heiß ist, als dass sie auszuhalten wäre, modelliert wird, einhergehend mit einer sich im Verlauf der Zeit vollziehenden Steigerungsdramaturgie. Und mit der Inszenierungsweise sprachlicher Äußerungen wird auch nochmal auf das Rhizomatische des Metaphorisierens zurückzukommen sein, wie es als typisch für DER KRIEGER UND DIE KAISERIN postuliert wurde.

7 Vgl. für eine Metaphernanalyse jener sich über zwölf Minuten ziehenden sechsten Szene Kappelhoffs Ausführungen hierzu in Müller/Kappelhoff: Cinematic Metaphor und Kappelhoff: Kognition und Reflexion. 
Das erste Mal artikuliert sich die Wahrnehmungserfahrung eines solchen Soges mit der Szene, die Sissis erste Nacht daheim nach ihrer Rückkehr aus der Unfallklinik zeigt (neunte Szene, erste Ausdrucksbewegungseinheit: 0:34:46-0:36:53, Abb. 30 ${ }^{\star}$. Sissi ist zu sehen, wie sie in ihrem Zimmer am Schreibtisch sitzt. Langsam, aber stetig zoomt die Kamera an sie heran, und aus dem Off spricht ihre Stimme die Worte eines Antwort-Briefes an Meike:

Sissi
„Wenn ich doch nur nicht immer an den Mann denken müsste.“

Erst zum Ende der Ausdrucksbewegungseinheit wird man wieder zu Sissi zurückkehren. Man wird sehen, wie sie, im Bett liegend, plötzlich schwer atmend aus dem Schlaf fährt. Dazwischen - in der Passage zwischen den Worten der verliebten Sissi und ihrem aufgewühlt-plötzlichen Erwachen - entfaltet sich das mentale andauernde Gebundensein eines ,Immer-an-jemanden-denken-Müssens ‘ als eine den physischen Körper bedrohende, horrorhafte Sogerfahrung. Traum und Trauma zeigen sich hier mit- und ineinander.

Noch während Sissis Stimme zu hören ist, wechselt die Einstellung und zeigt nun das im Dunkeln liegende Zimmer von Walter und Bodo. Das fast vollständige Schwarz des Bildfeldes ist durchgängig immer wieder durchwirkt von tiefem Grollen, krachendem Donner und dem gleißenden Licht von Blitzen. Auf einem dumpfen, tiefen Dröhnen, das im weiteren Verlauf ganz langsam anschwillt, liegt eine hohe, später sich um eine Oktave senkende, sirrende Tonlinie, die zwischen wenigen nah beieinander liegenden Tönen schwankt. Der Raum, in dessen nur von Blitzen durchzuckter Dunkelheit sich der Zoom fortsetzt, wird hier raumlos. Mit dem Klang dehnt sich eine Fläche unbekannten Ausmaßes aus, und damit wirkt der ganze audiovisuelle Raum dieses expressiven Musters noch wie ein gedunkeltes Ölgemälde der Romantik, in dessen Fläche man hineingesogen wird. Ein einziger schwacher Lichtfleck zeigt die beiden Brüder, schlafend. Mit geschlossenen Augen richtet sich Bodo ruckartig auf. In (durch geringe Tiefenschärfe) weicher Nahaufnahme ruht der Kamerablick auf seinem von Schweiß glänzenden Gesicht. Einer bewegungslosen Bewegung gleich, folgt die Kamera in schräger Aufsicht dem im Licht liegenden Körper, der sich nun - schlafend erhoben hat und mit langsamen Schritten durch das Dunkel wankt. Zusammen mit dem Sound realisiert sich der Sog nun erneut auch wieder durch die Kamerabewegung, wenn mit einem Einstellungswechsel der Blick an einen freistehenden schwarzen Ofen gleichförmig heranzoomt. Es ist ein Blick von hinten durch Bodos sich langsam und gleichförmig zum Ofen vorwärts schiebende Beine hindurch. Bildkompositorisch im Zentrum des Bildfeldes, dringt das glühende Licht des 

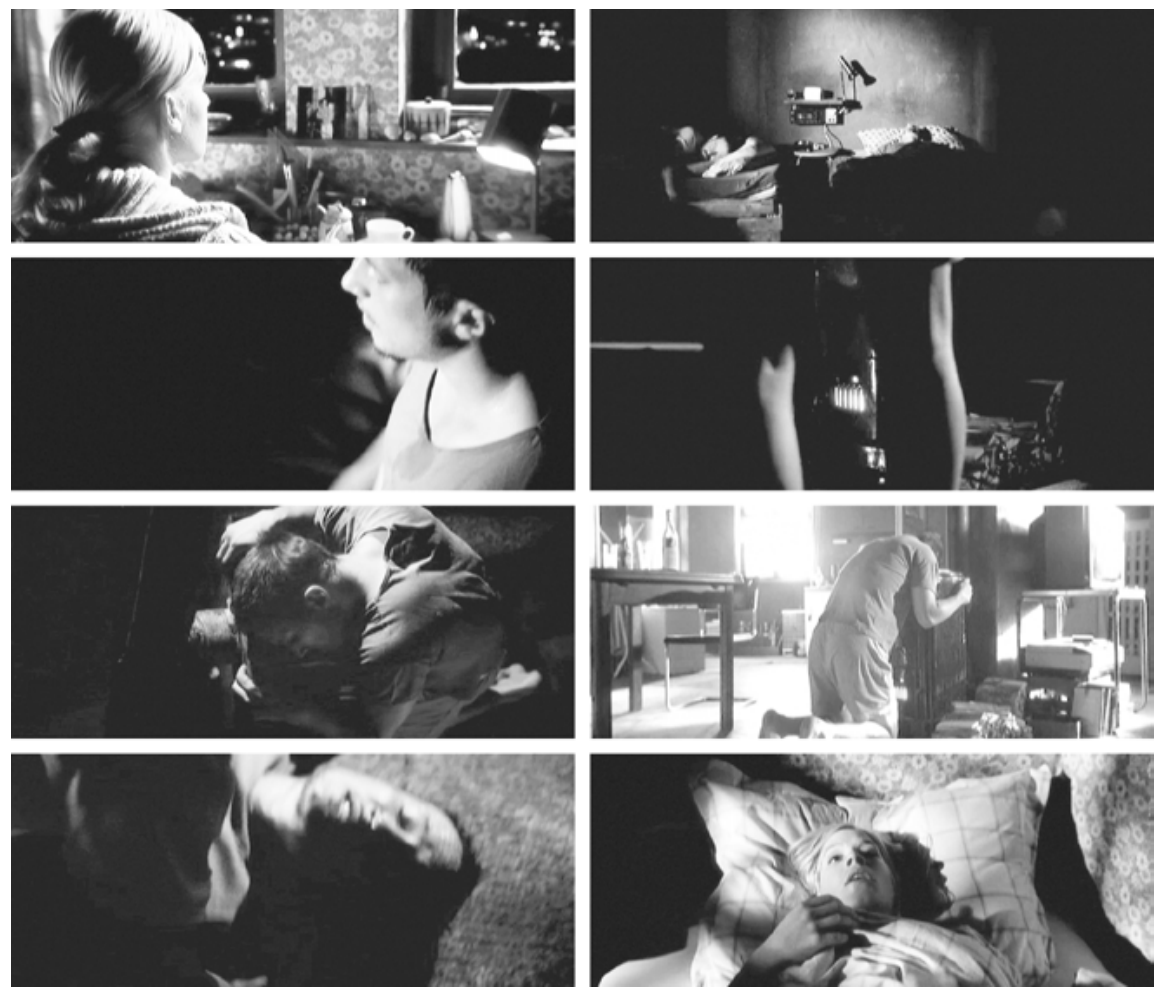

Abb. 30: Das Zusammenspiel von Kamerabewegung, Bildkomposition und Sound evoziert den Ausdruck einer gedehnt-soghaften, sich in ihrer Spannung intensivierenden Bewegung, die zuletzt konterkariert wird (DER KRIEGER UND DIE KAISERIN, neunte Szene, erste Ausdrucksbewegungseinheit: 0:34:46-0:36:53). ( $\rightarrow$ Abb. 30*, Farbbogen)

brennenden Feuers durch Ofenschlitze grellorange leuchtend in die Dunkelheit. Mit dem nächsten Einstellungswechsel sieht man Bodo, wie er sich langsam vor den Ofen kniet. Bodo ist dem Ofen jetzt mit dem ganzen Körper ganz nah, seine Hände umschließen den Ofenkörper, und sein Gesicht legt sich oben auf den Ofen drauf. Das Verdampfen seines Schweißes gleicht aufsteigendem Rauch. Und noch weiter dauert der Sog an. Der langsame und gleichförmige Zoom in einer Aufsicht zieht den Blick weiter in diese Umarmung eines brennenden Ofens hinein, die sich im ansonsten kontur- und grenzenlosen Schwarz des Bildes abzeichnet. Und im Sound hebt ein dumpfes, langsames, gleichmäßiges Schlagen an.

Erst jetzt wird dieser gedehnt-langsame, sich in seiner Spannung noch intensivierende Sog konterkariert werden. Mit dem Umschnitt auf das Gesicht des schlafenden Walter, der irgendwann die Augen öffnet, dauert der Zoom zunächst noch für Weile fort, doch schon hier kehrt die Tiefenschärfe zurück ins zoomende 
Bild, das noch einmal den Ofen umklammernden Bodo zeigt, bevor dann ein audiovisuelles Staccato einsetzt. Mit dem lauten Ausstoßen des Namens seines Bruders erhöht sich die Schnittfrequenz, Walter reißt seinen Bruder vom Ofen weg und wirft ihn auf den Boden, und mit seiner ganzen Kraft hält er ihn fest. Die miteinander kämpfenden Körper zucken, Bodos Stoßatmen presst und zieht Luft durch die Zähne, wild und verzweifelt schmerzstarrend reißt er seine Augen weit auf. Und Sissi schreckt schwer atmend aus dem Schlaf.

\section{,Traum \& Trauma II‘: dreizehnte Szene, 0:51:05-1:04:48}

Fünfzehn Filmminuten später: Sissi hat Bodo zwischenzeitlich wiedergefunden, er allerdings erwidert ihren Wunsch des Wiedersehens nicht. Von ihm brachial zurückgewiesen - sie berührte ihn sanft an der Schulter, er schlug sie zu Boden ist Sissi in die Klinik und auf ihr Zimmer zurückgekehrt. Doch sie wird noch in der Nacht erneut zu ihm gehen - und erneut zurückgewiesen werden.

Am Anfang und Ende dieser fast vierzehn Minuten dauernden Szene des erneuten Wiedersehens und der erneuten Zurückweisung (dreizehnte Szene) stehen jeweils Liebespaare: Liebespaare, die auf unterschiedliche Weise mit intensiver Wärme und Hitze korrelieren. Es sind Liebespaare, bei denen die filmische Expressivität, mit der sie zur Wahrnehmung gebracht werden, die Ausdrucksqualität eines letztlich konterkarierten Sogs variieren und wiederholen, wie sie soeben mit Bodos Schlafwandeln in einer Gewitternacht beschrieben wurde.

Zwischen den zwei Liebespaar-Inszenierungen, die im Weiteren skizziert werden, liegt (unter anderem) ein Dialog zwischen Sissi und Walter. Bodo, so Walter, sei nichts für Sissi, und sie, weil sie eine Frau sei, wäre für Bodo „ein Problem zuviel“. Sissis Frage, ob Bodo „was gegen Frauen“ habe, bejaht er -

Walter

„[...] weil seine tot ist.“

Sissi

„Seine Frau? Ach so. Warum ist sie tot?“

Walter

„War so'n Unfall auf 'ner Tankstelle, was geht dich das an?“

Sissi

„Nichts.“

Walter

„Bodo war auf'm Scheißhaus. Sie hat getankt und das Ding ist in die Luft gegangen, irgendwie. Bodo kommt vom Klo, alles weg, verbrannt, die Frau auch.“ 
Und so ist - in aller nur erdenklichen sprachlichen Schlicht- und Nüchternheit von Frage und Antwort - mit Bodos bei einer Explosion verbrannten Frau auch hier von einem Liebespaar die Rede, das in Korrelation steht mit extremer Hitze. Mit einer Hitze, die in ihrer Verletzung todbringend, allvernichtend ist.

Diesem Dialog vorgelagert ist mit dem Szenenbeginn das eingangs bereits erwähnte Filmschauen Sissis (dreizehnte Szene, erste Ausdrucksbewegungseinheit: 0:51:05-0:52:02, Abb. 31) ${ }^{\star}$, in dessen Anschluss sie sich wieder auf den Weg zu Bodo machen wird. Jener Szenenanfang ist in einer Weise inszeniert, die ihn ins Verhältnis zu der oben analysierten gewitternächtlichen Schlafwandelsequenz treten läßt - nimmt er doch für Berührungen des Küssens Aspekte von dessen Inszenierungsweise in sich auf, um sie zugleich entscheidend zu modifizieren.

Zusammen mit Sissi, der niedergeschlagenen Abgewiesenen, schaut man auf einem alten kleinen Röhrenfernseher die deutsche Synchronfassung von de Sicas MIRACOlo a MILANo: In dem zu sehenden Ausschnitt ${ }^{8}$ küsst ein junger Mann mit Energie ein Mädchen, und das Mädchen küsst ihn genauso energiegeladen zurück - beide Gesichter strahlen vor Glück. Und als er sie fragt, „Willst du die Sonne?“, und sie dies bejaht, geht zuletzt am Horizont die Sonne auf. Die durchgängig erklingende de-Sica-Filmmusik (mit Zitter und Streichorchester) mit ihrem gedehnten, immer höher wandernden Seufzermotiv schwillt hier zu einem strahlenden Klang an und erreicht ihren Höhepunkt. Das extreme Blau des Fernsehbildes (das hier visuell zuvorderst mit dem Blau eines großen Bildes an Sissis Zimmerwand korrespondiert, das einen mit weiten Schwingen fliegenden Vogel zeigt) widerstrebt dabei noch der Wahrnehmung der im Fernsehbildfilm beschworenen Sonne als einem heißen Himmelskörper. Doch zugleich breitet sich ein Gelb mit der Einrichtung von Sissis Zimmer - mit der gelben Tapete, der gelben Bettdecke - großflächig im Bild aus. Und auch ist alles immer wieder von gleißend hellem Blitzlicht überzogen, Donner kracht und grollt: Es ist erneut eine Gewitternacht. Geradlinig führt eine Point-of-View-Montage, die einhergeht mit einem langsamen Zoom, im mehrfachen Hin-und-Herwechseln Sissis Gesicht dem Fernsehbild immer näher zu, bis die Betrachtung vom Liebespaar und der aufgehenden Sonne das gesamte Bildfeld einnimmt. Ein letztes Mal wechselt die Großaufnahme des Fernsehbildes zurück zur Großaufnahme von Sissi. Hier hat das expressive Muster einer langgezogenen, soghaften, von Explosionen durchzogenen Annäherung, die durch Musik und Schauspiel (im Fernsehbild) zugleich mit einer ganz anderen Energie durchzogen ist, seinen Umschlagspunkt: im Aufstehen Sissis und ihrem Verlassen des Bildfeldes, als die Musik auf ihrem

8 Der Ausschnitt, wie er hier in Tykwers Film zu sehen ist, ist eine verdichtende Bearbeitung von der Originalsequenz bei de Sica. 
höchsten Punkt stehen geblieben ist und Leere zurücklässt. Zu Anfang der Szene des erneuten Wiedersehens und der erneuten Zurückweisung ist so ein Bild der Sehnsucht entstanden, das hier jedoch nicht verbrennend ist.
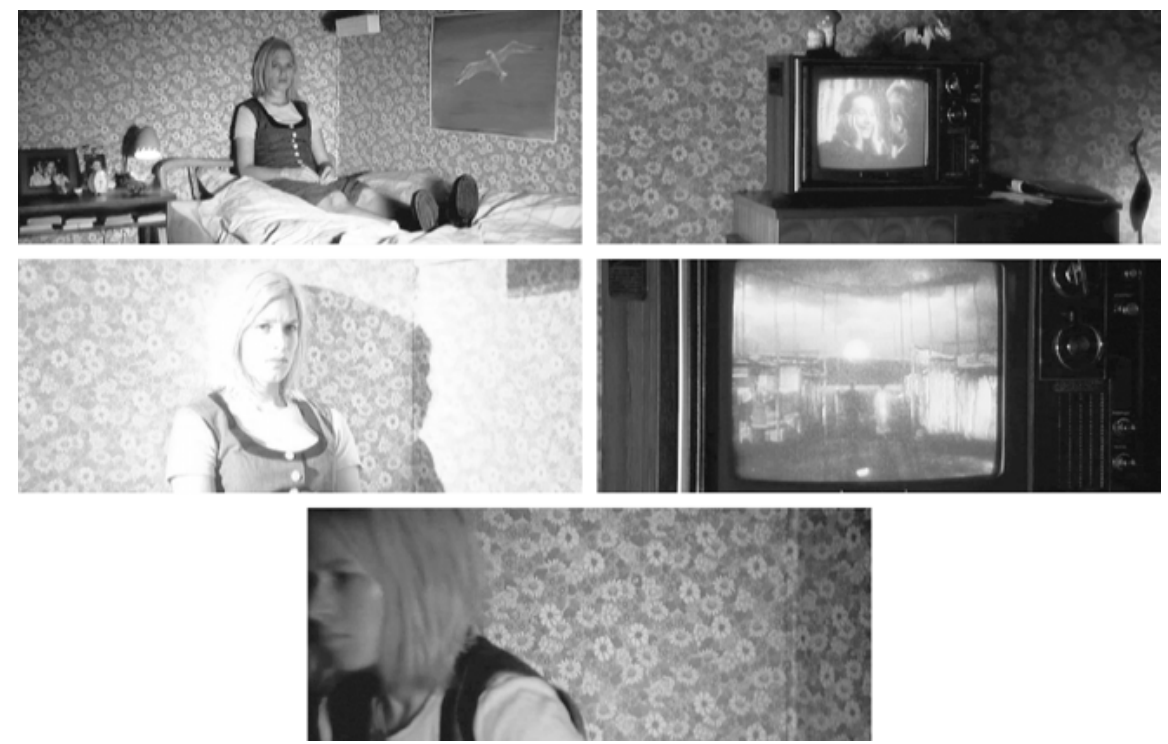

Abb. 31: Ein Bild der Sehnsucht: Das Zusammenspiel von Kamerabewegung und Montage evoziert den Ausdruck einer zuletzt abbrechenden gedehnt-soghaften Bewegung, die durch Musik und Schauspiel (im Fernsehbild) jedoch zugleich mit anschwellender, kraftvoller Energie durchzogen ist (DER KRIEGER UND DIE KAISERIN, dreizehnte Szene, erste Ausdrucksbewegungseinheit: 0:51:05-0:52:02). ( $\rightarrow$ Abb. 31*, Farbbogen)

Zum Szenenende hingegen kehrt das Ausdrucksbewegungsbild des zum Schluss konterkarierten Sogs in seiner dunklen, horrorhaften Variante wieder (dreizehnte Szene, letzte Ausdrucksbewegungseinheit: 01:02:06-01:04:48, Abb. 32) ${ }^{\star}$, eine halbe Stunde, nachdem man als Zuschauer ein solches erstmalig durchlaufen hat. Die Szene endet im Zimmer von Bodo und Walter, wo Bodo duscht, nachdem er Sissi wieder von sich gestoßen hatte. Hier wird sich auch der LiebestraumStatus des Bewegungsbildes variierend wiederholen: Denn der im Folgenden beschriebenen Sequenz unmittelbar voran geht eine Großaufnahme Sissis, die unter freiem Himmel mit offenen Augen auf einer Wiese niedergesunken ist. Ihr Gesicht blendet über in ihren Point-of-View: ein schwarzer, das ganze Bildfeld füllender, von Sternen übersäter Himmel. Ein langsamer Zoom beginnt, und in den Himmel blendet sich ein überirdisch erscheinendes Bild Bodos: Bodos nackter Körper hinter den Scheiben einer (durch die Überblendung noch zunächst freischwebenden) Duschkabine. Nun beginnt die letzte Ausdrucksbewegung der 

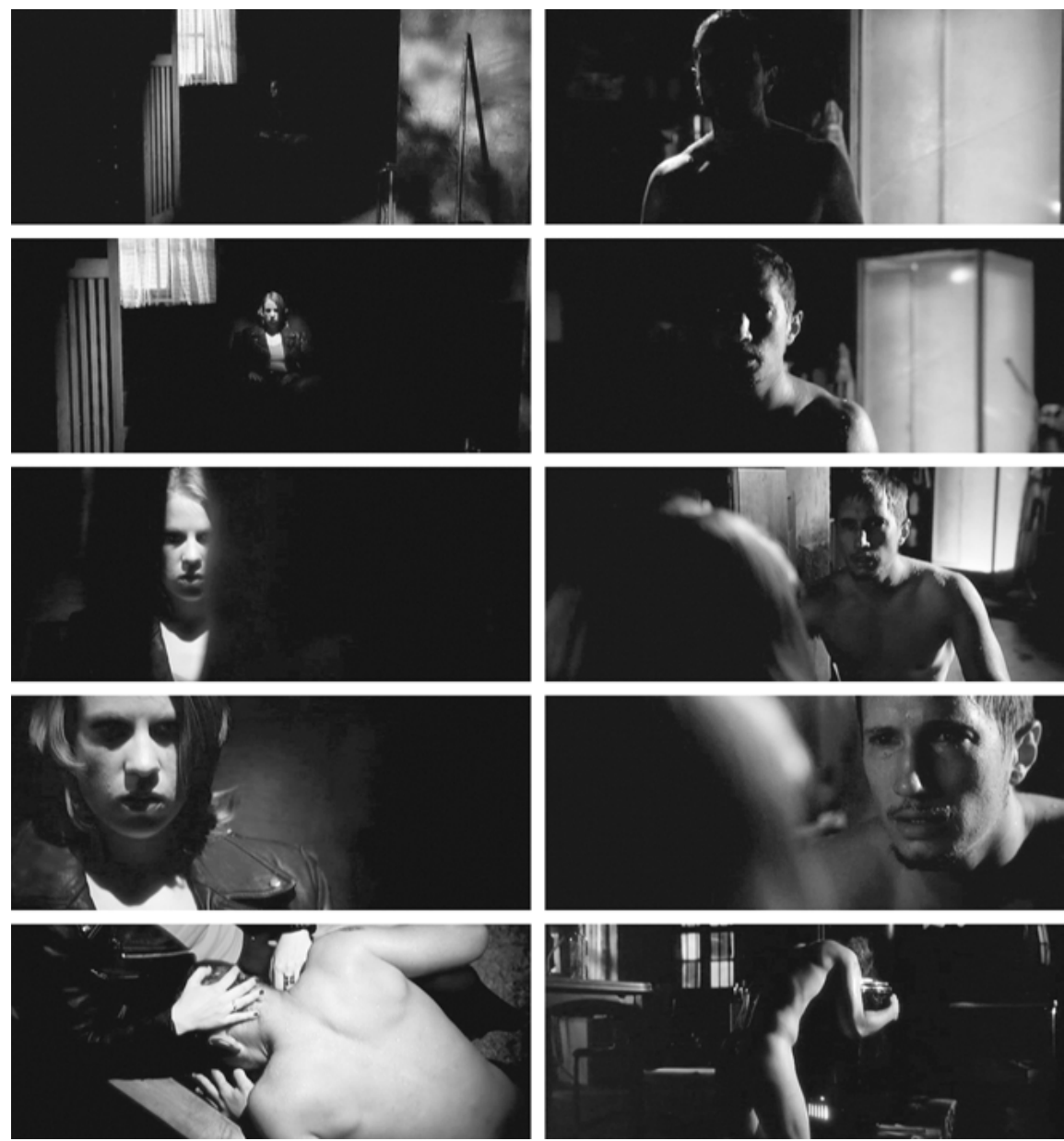

Abb. 32: Bildkomposition, Montage, Kamerabewegung und Sound evozieren den Ausdruck einer gedehnt-langsamen, angespannte geradlinigen Sogbewegung, die zuletzt konterkariert wird um dann doch neu anzusetzen. (DER KRIEGER UND DIE KAISERIN, dreizehnte Szene, letzte Ausdrucksbewegungseinheit: 01:02:06-01:04:48. ( $\rightarrow$ Abb. 32*, Farbbogen) 

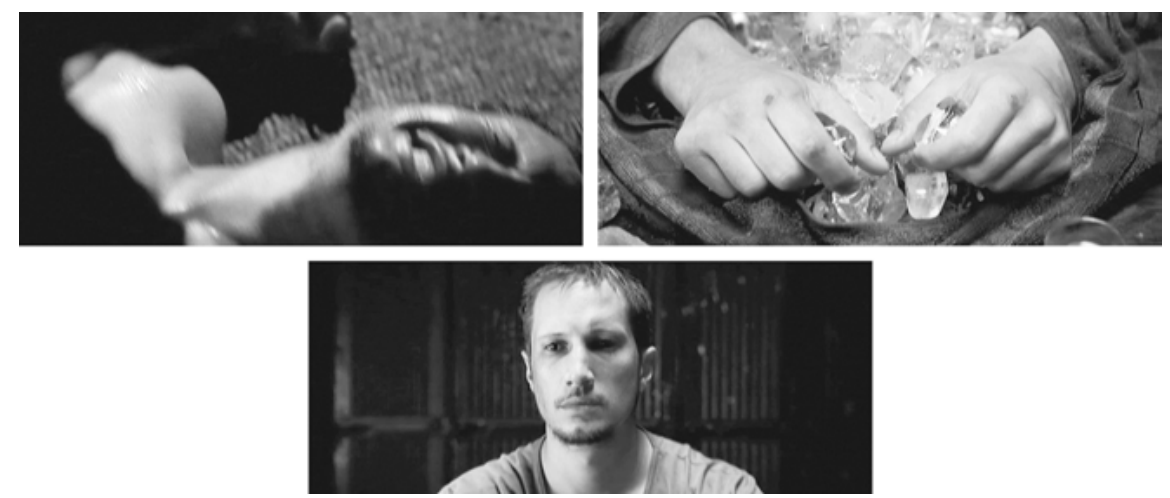

Abb. 32 (Fortsetzung)

Szene, in deren entstehendes Bild Sissis Stimme zuletzt eindringen wird, flüsternd, wiederholt Bodos Namen aussprechend: Im Zusammenspiel von Bildkomposition, Montage und Sound kommt die Wahrnehmung einer gedehnt-langsamen, unter Spannung stehenden geradlinigen Sogbewegung, die zuletzt konterkariert wird, zum Ausdruck.

Erneut drängt dabei ein Schwarz, das sich im nur punktuell ausgeleuchteten Bild ausbreitet, die Räumlichkeit immer wieder zurück, und auch ansonsten ist das Bild ein Widergänger -mit einigen Variationen: Eine halbe Stunde zuvor noch gab es den Blick auf Bodos schweißnassen, durch das dumpfe Dröhnen und hohe Sirren einer Gewitternacht schlafwandelnden Körper. Dies ist nun der Blick auf einen wachen, eben aus einer leuchtenden Duschkabine gekommenen gehenden Körper, von dem man noch das Wasser herabtropfen hört, hinein in eine schweigende, schwarze Stille, die von einer tiefen, schnellen, angespannt-gleichförmigen Schwingung durchzogen ist. Was dort noch ein Blick durch Bodos Beine auf das grellorangene glühende Feuer des Ofens war, ist nun der Blick auf das stumme Gesicht einer jungen Frau in schwarzer Lederjacke und grellpink leuchtendem Shirt, die im Schwarz des Bildes versinkt - ein Gesicht ohne Augen, nur mit schwarz verschatteten Höhlen. Dort zeigte eine Zoom-Aufsicht noch, wie Bodo den Ofen umfasst und sein Gesicht auflegt. Nun zeigt eine solche Einstellung, wie Bodo weinend mit seinem Gesicht im Schoß dieser Frau liegt, die auf Bodos Frage, wo Walter sei, nur stumm und langsam den Kopf geschüttelt hat. Ruhig streichelt sie über Bodos Nacken, und mit dem gleichförmigen Zoom nähert sich der Blick dem nackten, weinenden Körper immer mehr an. Der gedehntlangsame Sog zeigt sich hier mit zwangsläufiger Geradlinigkeit: mit einer Pointof-View-Montage von Bodos langsamem, gleichförmigen Gehen, verbunden mit langsamen, gleichförmigen Kamerafahrten bis zu den Schuss-Gegenschuss-Groß- 
aufnahmen der Frau und Bodos sowie dem Aufsicht-Zoom, in der dumpfen Stille einer tiefen, schnellen, unter Spannung stehenden gleichförmigen Schwingung.

Dann setzt das Staccato ein und die Montage gleicht sich wieder ihrer Ursprungsfassung an: Bodo, vor dem brennenden Ofen kniend, mit aufliegendem Gesicht; Walter, wie er -nachdem hier Sissis Flüstern zu hören war - laut Bodos Namen ruft und seinen Bruder vom Ofen wegreißt. Allein was vormals in diesem den Sog konterkarierenden Staccato noch ein durch die Zähne gepresstes Stoßatmen Bodos war, ist jetzt schon lauter, intensiviert sich, ist ein unterdrücktes Weinen und Schluchzen. Das Bewegungsbild schließt zuletzt mit dem Blick auf Bodo: mit Eiswürfeln in seinen Händen, stumm am Tisch sitzend neben Walter. Es verbindet sich auf diese Weise sinnlich mit dem Anfang der Szene (dem rechteckigen blauen Fernsehbild) wie mit dem Anfang des Films (dem Eiswürfel in Sissis Hand) - doch mit einem langsamen, isolierenden Zoom, der den Raum um Bodo erneut verschwinden lässt, ist es ein Loop-haftes Ende, in dem der Sog noch wiederkehrt.

Wieder artikuliert sich im filmisch modellierten Wahrnehmen und Fühlen das Verstehen eines Liebestraums, eines Denkens an einen geliebten Menschen, einer Sehnsucht: als eine den physischen Körper bedrohende, horrorhafte Sogerfahrung einer zu heißen Berührung, zu heiß, als dass sie auszuhalten wäre noch die Wiederholung selbst ist Teil dieser Sogerfahrung. Und mit der gesamten Szene vereindeutigt sich dieses Denken als metaphorisches Wahrnehmungsszenario eines Liebestraumas - mit der de Sica-Liebesszene, deren Sehen von Gewitterexplosionen durchzogen ist, mit der vorangegangenen Rede Walters über Bodos bei einer Explosion verbrannten Frau, und mit dem oben beschriebenen Prozess der Ersetzung des brennenden Ofens durch eine Vision dieser Toten.

\section{,Ein neues Trauma': achtzehnte Szene, 1:24:17-1:32:07}

Eine letzte Sequenzanalyse zu DER KRIEGER UND DIE KAISERIN bringt nun die exemplarischen Betrachtungen zum Metaphorisieren beim Spielfilm zum Abschluss. Hierfür wird, mit dem Beginn einer späteren Szene, noch die nächste Entwicklung betrachtet, die sich in dem hier ausschnitthaft untersuchten Zusammenhang filmischen Metaphorisierens mit Blick auf das Erleben des Sogs einer zu heißen Berührung und dessen Korrelation mit dem Erfahrungsbereich von Liebe vollzieht. Mehrere Verschiebungen finden dort statt: Es geht nicht mehr um die Liebe zwischen Mann und Frau, sondern um die zwischen Brüdern. Die Aktivierung des $\mathrm{Zu}$-Heiß, des Verbrennens wird sich nun wieder sprachlich vollziehen. Nicht mehr Traum und Trauma werden sich ineinander zeigen, nicht mehr Sehnsucht, sondern eine erneute traumatische Erfahrung: Zwanzig Filmminuten nach der zuletzt 
analysierten Szene kommt Bodo auf Sissis Station der geschlossenen psychiatrischen Anstalt an und wird dort vom Tod seines Bruders erfahren (achtzehnte Szene, erste Ausdrucksbewegungseinheit, 1:24:17-1:27:02, Abb. 33). (Bodo war von Sissi zuvor bei sich auf ihrem Zimmer versteckt worden, nachdem Bodos und Walters Bankraub scheiterte und Walter angeschossen wurde. Sissi, deren Weg sich mit jenem Bodos bei der Bank erneut kreuzte, konnte ihm nun zu Hilfe kommen und Walter noch mit ins Krankenhaus bringen.)

In jener Sequenz nun, deren Handlungsraum der Gemeinschaftsraum der Station ist, den Bodo betritt, wird viel und in drei parallelen Konstellationen gesprochen: Es gibt ein gruppentherapeutisches Gespräch, Bodo spricht mit einem Patienten, und darüber hinaus läuft ein Fernseher. Im Lauf der Zeit transformiert sich ein ruhiges Sprechen dabei in eine Vielstimmigkeit, und schließlich mündet alles in einem nicht enden wollenden Schreien. Im Ganzen entfaltet sich mit der Sequenz erneut eine variierende Wiederholung einer soghaften Bewegung, die am Ende konterkariert wird. Denn diese stimmlichen Artikulationen fügen sich zum nun schon vertrauten Muster einer langsamen, gleichförmigen Kamerabewegung, die sich mit einer Variante der schon für vorherige Ausdrucksbewegungen beschriebenen geradlinigen gleichförmigen Point-of-view-Montageform verbindet. Bodo wird sich (parallel zu Sissi in der Szene des Filmschauens) kontinuierlich dem Fernsehbild annähern, bis dieses schließlich das gesamte Bildfeld füllt und der Umschnitt Bodos Gesicht zeigt, in Großaufnahme. Eine sich steigernde Anspannung durchzieht das expressive Muster, nicht zuletzt aufgrund des immer starrender werdenden Blicks Bodos auf den Fernseher sowie der angespannten Blicke Sissis und anderer Patienten aus der Therapierunde. Und es wird schließlich Bodos anhaltendes Schreien, Toben und Zerstören des Fernsehers sein, es werden die ihn zu Boden ringenden Pfleger sein, es werden die gesteigerte Schnittfrequenz und wechselnden Einstellungsperspektiven sein, die den Sog mit aller Kraft konterkarieren.

Wie die verschiedenen Sprechkontexte in dieser Sequenz durch Montage und Sounddesign inszeniert sind, wie die vielfältigen Sprechakte durch Taktungen und Lautstärken miteinander komponiert sind, sei nun genauer betrachtet (vgl. auch Abb. 34 am Ende der Analyse). Denn nicht nur die stimmliche Ausdrucksqualität ist für das Metaphorisieren von Belang (insofern sie eben Teil des filmischen Bewegungsmusters ist), sondern auch die damit zum Ausdruck gebrachten Worte. Das Sprechen ist in sehr konkreter Weise bedeutsam, geht es doch ein in über Szenengrenzen hinausreichende Wahrnehmungsszenarien. Und nicht zuletzt zeigt sich in der Inszenierungsweise der sprachlichen Äußerungen pars pro toto auch die rhizomatische Figuration des Metaphorisierens - die dynamische Form des sich Verzweigens und -Verbindens also, mit der der Prozess der Bedeutungskonstruktion beschrieben werden kann, wie ihn DER KRIEGER UND DIE 

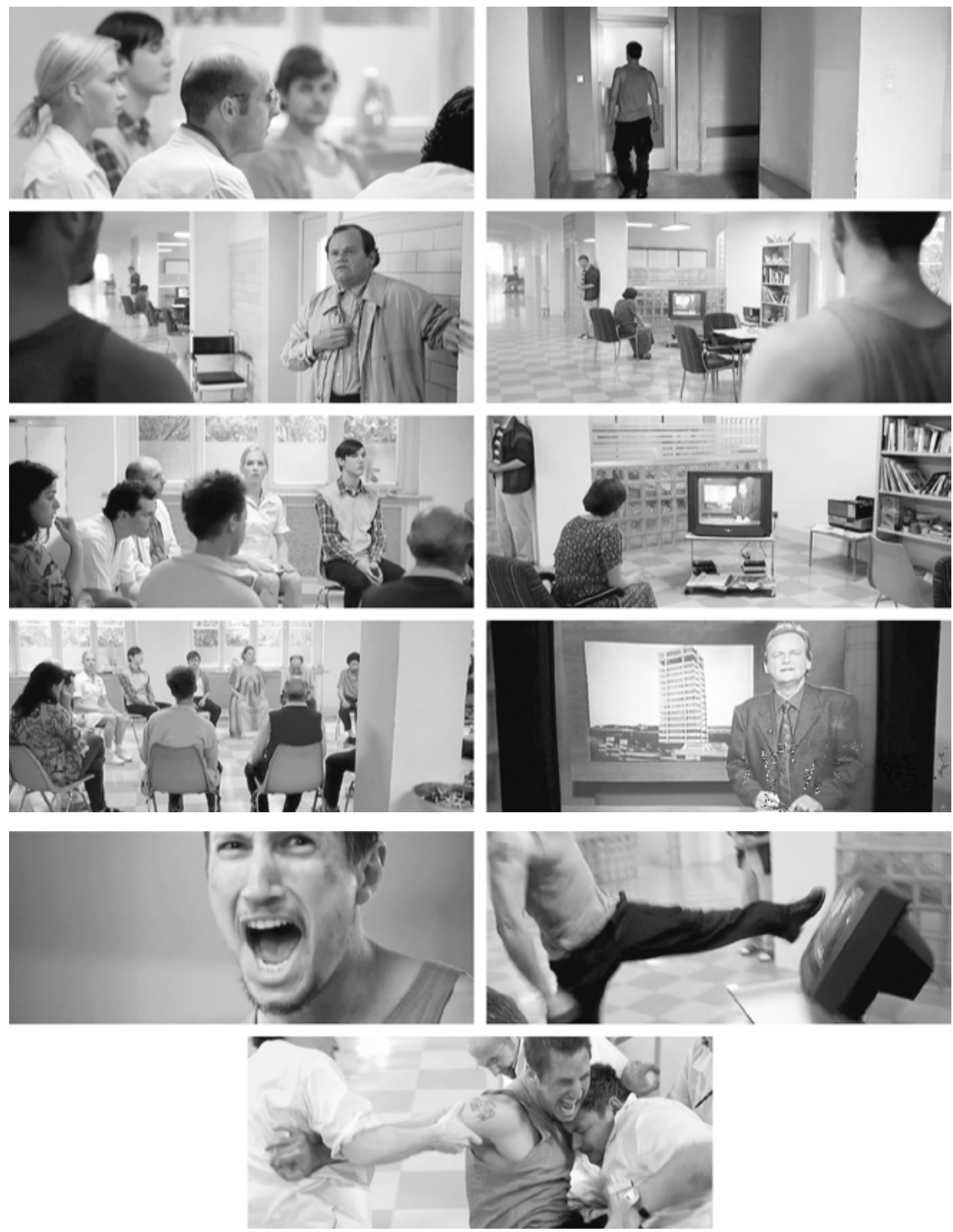

Abb. 33: Im Verlauf der Zeit transformiert sich ein ruhiges Sprechen in eine unruhige Mehrstimmigkeit und mündet schließlich in einem Schreien. Im Zusammenspiel mit Montage, Kamerabewegung und Schauspiel evoziert dies einen gedehnt-langsamen Sog, dessen sich steigernde Anspannung sich zuletzt explosionsartig entlädt. (DER KRIEGER UND DIE KAISERIN, achtzehnte Szene, erste Ausdrucksbewegungseinheit: 1:24:17-1:27:02.) 
KAISERIN im Ganzen modelliert und sich darüber noch $\mathrm{zu}$ diesem eigenartigen Liebesmärchen fügt. Wo sich noch alles mit allem verbindet, obwohl es doch ganz unterschiedliche Kontexte hat.

Die Sequenz beginnt ruhig und konzentriert im Gemeinschaftsraum der Psychiatriestation mit einem Gruppentherapiegespräch. In Nahaufnahmen sieht man zunächst den Chefarzt - neben sich Kollegen, Sissi und Patienten - wie er die Gesprächsrunde eröffnet.

\footnotetext{
Oberarzt

„(...) Wir sind hier alle ein bisschen angespannt im Moment. Wir müssen auch über die Probleme reden. Ich hab Sissi ausdrücklich gebeten, heute wieder dabei zu sein. Möchte jemand etwas zu diesem Thema sagen?“
}

Die langsame, stark abgeflachte Kreisbewegung der Kamera, die parallel zu diesen Worten anhebt, setzt sich nahtlos und gleichförmig fort, wenn ein Einstellungswechsel erfolgt und mit einem anderen Ausschnitt der großen Patientenrunde gezeigt wird, wie der Patient Walter das Wort ergreift.

\section{Patient Walter}

„(...) Also, ich wollt mal so sagen. Zu dem Problem, also so im Ganzen. ... “

So klar die Ansprache des Oberarztes im Ton ist, so unklar bleibt, um was für ein Thema, um was für ein Problem es hier genau geht. Weder wird es vom Arzt direkt spezifiziert, noch hilft die Antwort des Patienten weiter. Der redet weiter, stammelnd, in Satzfetzen, mit Pausen und Wiederholungen, ohne irgendetwas zu erklären:

\section{Patient Walter}

„Das Problem ist ... das hat zu tun mit ... das Problem ist ... also vor allem ... das hat zu tun mit ...“

Doch hier hat jetzt die Einstellung schon gewechselt. Man hört dieses Stammeln zwar weiterhin, aber es ist nun ein Hintergrundgeräusch. Bodo ist zu sehen. Allein und desorientiert geht er durch einen Flur, öffnet eine Tür und betritt den offenen Gemeinschaftsraum, der sich nun nach und nach mit diversen Geräuschen zu füllen beginnt und so die anfängliche konzentrierte Atmosphäre diffus werden lässt: Die Tür schlägt laut hinter Bodo zu, man hört mehrere Patienten ihre gewohnten Flüche ausstoßen; ein klimpernder Fernsehjingle erklingt und die schleifenden Schritte von Bodo hallen. Während Bodo immer weiter - langsam, aber ohne auch nur einen Moment innezuhalten - den Flur entlang geht und das Gewirr von aus dem Fernseher dringender Musik und dem Reden der Therapierunde dichter wird, wird Bodo von einem weiteren Patienten direkt angesprochen. Ein kurzer, grotesker Wortwechsel entspinnt sich: 
Patient (2)

„Was bist du denn für einer? He? Was bist du denn? Bist du was?“

Bodo

„Wo, wo bin ich'n hier?“

Patient (2)

„Ja, is' klar, is' klar. Du gehst woanders hin, klar, logisch. Is' klar. Ins Krematorium, ne? Ins Krematorium. Bleibt nix übrig. Weg ist weg.“

Der Patient redet weiter, blickt dem weitergehenden Bodo hinterher. Allerdings rückt nun erneut etwas anderes akustisch in den Vordergrund, jetzt ist aus dem Fernseher der Nachrichtensprecher zu verstehen, wie er vom Banküberfall des vorherigen Tages berichtet.

\section{Nachrichtensprecher}

„(...) Die Täter hatten einen Tunnel zum Keller der belieferten Bank gegraben und die Geldboten bei der Auslieferung überrascht.“

Bis zu diesem Moment war es die gleichbleibende Langsamkeit des Kamerabewegungsflusses, der eine Kontinuität zwischen den verschiedenen Redekontexten stiftete, die in diesem offenen Gemeinschaftsraum zusammenkommen (und zwischen denen die Kamera auch im weiteren Verlauf wechselt, ohne ihren Bewegungsfluss zu unterbrechen): das Therapiegespräch (gestaltet mit einer runden Form) und das Sprechen von Bodo und den Patienten auf dem Flur sowie die Nachrichtenstimme aus dem Fernsehen (gestaltet mit einer geraden Form). Jetzt intensiviert sich diese Verschränkung der Redekontexte noch durch das Sprechen selbst. Denn die Atempause des Nachrichtensprechers wird von einem Wort gefüllt, das im Therapiegespräch fällt -

Oberarzt

„(...) Liebe (...)“

- bevor dann sogleich die Nachrichtenstimme weiterspricht -

Nachrichtensprecher

„Ein Wachpolizist schoss auf die fliehenden Diebe und verletzte einen von ihnen schwer.“

- und erneut das Therapiegespräch zu hören ist:

Oberarzt

„Von welcher Liebe reden Sie denn? Sagen Sie doch. Ich find das sehr interessant.“

Und so ist es nicht nur die Taktung, sondern auch der sich reimende Klang der Worte „Liebe“ - „Diebe“ - „Liebe“, weshalb dieser offene, immer komplexer 
werdende Gemeinschaftsraum mit seinen multiplen Zentren im Sehen und Hören ein Wahrnehmungsraum ist.

Mit dem Erreichen der Großaufnahme des Fernsehers richtet sich schließlich alle Aufmerksamkeit auf die dort verkündete Todesnachricht -

\section{Nachrichtensprecher}

„Der 40-jährige Mann erlag jedoch kurze Zeit später den Verletzungen.“

- und dann verdrängt Bodos Schreien und körperliches Wüten all das vielstimmige Sprechen, das eben noch diesen Wahrnehmungsraum füllte.

Handlungs- und figurenpsychologisch betrachtet, verweigert das wirre, zwanghafte Sprechen der Psychiatriepatienten - das Stammeln und das zwanghafte Reden über Krematorien (oder auch, wie in der früheren Tanzsequenz, das obszön-fäkale Fluchen) - das Gespräch: Das Stammeln antwortet dem Chefarzt nicht auf die Aufforderung, dass man doch nun mal bitte auch über die Probleme reden solle. Die Replik, er gehe logischerweise ins Krematorium, beantwortet nicht Bodos Frage, wo er denn hier sei (die Antwort auf diese Frage wird erst mehrere Filmminuten später, zum Ende der Szene, durch den Chefarzt erfolgen). ${ }^{9}$ Und dass der Chefarzt mit diesen Psychiatriepatienten ein Gespräch über Liebe führen kann, kann bezweifelt werden. Handlungslogisch gesehen gibt es hier also kein wirkliches Miteinander-Reden, keinen Dialog.

Vom hier eingenommenen Standpunkt filmischer Expressivität stellt sich dies jedoch anders dar. Denn es ist hier ein filmisches Bewegungsbild gegeben, das noch Teil eines großen Ganzen ist, welches dieses Reden von Problemen, vom Krematorium, von der Liebe und vom Tod wahrnimmt. Denn es ist der sich im Sehen und Hören von DER KRIEGER UND DIE KAISERIN vollziehende, sich mit Liebe korrelierende variierte Ausdruck eines gedehnt-langsamen, zuletzt konterkarierten Sogs einer zu heißen Berührung, der ein solches Reden als unterschiedliche, verzweigte, Sprechkontexte wahrnimmt (ein therapeutisches Gespräch, ein Wortwechsel zwischen einem Traumatisierten und einem Patienten, eine Fernsehnachricht), diese aber zugleich in ein verkörpertes Wahrnehmungserleben überführt, sie miteinander verbindet. Ein Wahrnehmungserleben, das gemeinsam, mit vielen anderen filmischen Bewegungsbildern, ein Verstehen von Liebe hervorbringt als der Schwierigkeit sich zu berühren, ohne sich zu verletzten, ohne sich Schmerzen zuzufügen.

9 Vgl. zu diesem Szenenende, und wie dort der Erfahrungsbereich von Wasser und Eis weiter elaboriert wird, auch die instruierende Analyse von Wedel in Michael Wedel: Filmgeschichte als Krisengeschichte. Schnitte und Spuren durch den deutschen Film. Bielefeld: transcript 2011, hier S. 403-413. 


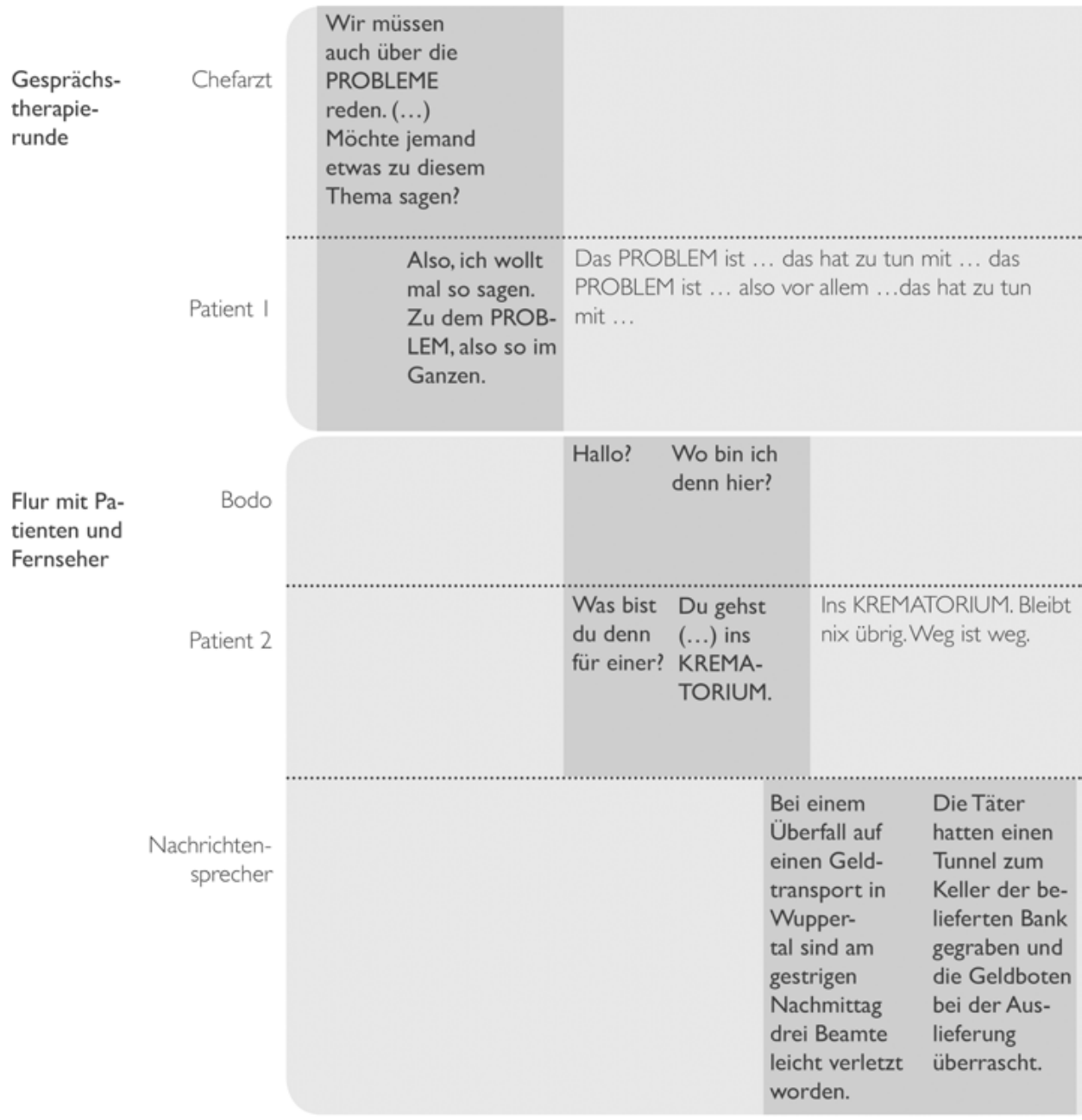

Abb. 34: Multiple Sprechkontexte, die durch Sound und Montage disparat bleiben und sich zugleich einheit). Großbuchstaben: Aspekte metaphorischer Erfahrungsfelder; dunkelgrau hinterlegt: akustisch im Hintergrund ist. 


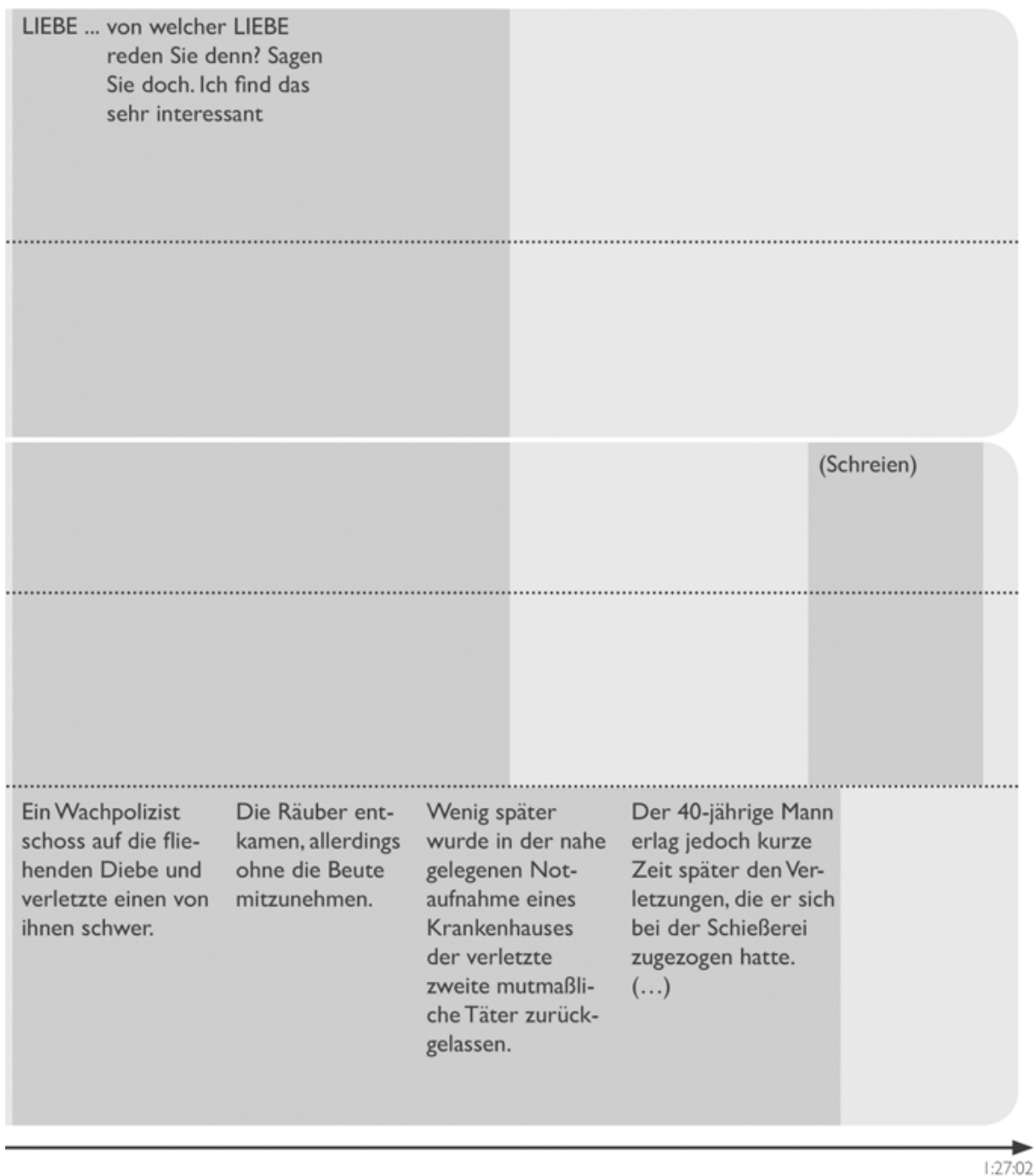

miteinander verbinden (DER KRIEGER UND DIE KAISERIN achtzehnte Szene, erste Ausdrucksbewegungsinszenatorisch evozierte Aufmerksamkeitsfelder. Feine Buchstaben: das Gesprochene, das 
